\title{
HL-LHC and ILC sensitivities in the hunt for heavy Higgs bosons
}

\author{
Henning Bahl ${ }^{1, a}$, Philip Bechtle ${ }^{2}$, Sven Heinemeyer ${ }^{3,4,5}$, Stefan Liebler ${ }^{6}$, Tim Stefaniak ${ }^{1}$, Georg Weiglein ${ }^{1}$ \\ ${ }^{1}$ DESY, Notkestraße 85, 22607 Hamburg, Germany \\ 2 Physikalisches Institut der Universität Bonn, Nußallee 12, 53115 Bonn, Germany \\ ${ }^{3}$ Instituto de Física Teórica, (UAM/CSIC), Universidad Autónoma de Madrid, Cantoblanco, 28049 Madrid, Spain \\ ${ }^{4}$ Campus of International Excellence UAM+CSIC, Cantoblanco, 28049 Madrid, Spain \\ ${ }^{5}$ Instituto de Física de Cantabria (CSIC-UC), 39005 Santander, Spain \\ ${ }^{6}$ Institute for Theoretical Physics (ITP), Karlsruhe Institute of Technology, 76131 Karlsruhe, Germany
}

Received: 25 June 2020 / Accepted: 14 September 2020 / Published online: 6 October 2020

(C) The Author(s) 2020

\begin{abstract}
The prediction of additional Higgs bosons is one of the key features of physics beyond the Standard Model (SM) that gives rise to an extended Higgs sector. We assess the sensitivity of the Large Hadron Collider (LHC) in the high luminosity (HL) run alone and in combination with a possible future International Linear Collider (ILC) to probe heavy neutral Higgs bosons. We employ the Minimal Supersymmetric Standard Model (MSSM) as a framework and assume the light $\mathcal{C P}$-even MSSM Higgs boson to be the Higgs boson observed at $125 \mathrm{GeV}$. We discuss the constraints on the MSSM parameter space arising from the precision measurements of the rates of the detected signal at $125 \mathrm{GeV}$ and from direct searches for new heavy Higgs bosons in the $\tau^{+} \tau^{-}, b \bar{b}$ and di-Higgs $(h h)$ final states. A new benchmark scenario for heavy Higgs searches in the $b \bar{b}$ channel is proposed in this context. For the future Higgs rate measurements at the HL-LHC and ILC two different scenarios are investigated, namely the case where the future rate measurements agree with the SM prediction and the case where the rates agree with the predictions of possible realizations of the MSSM Higgs sector in nature.
\end{abstract}

\section{Introduction}

The Large Hadron Collider (LHC) continues to measure the properties of the discovered Higgs boson [1,2] at $125 \mathrm{GeV}$ with increasing precision. So far, given the current experimental and theoretical uncertainties, the measurements are in good agreement with the SM predictions [3-6]. Nevertheless, in certain parameter regions, models with extended Higgs sectors often feature a SM-like Higgs boson that is compatible with the experimental data. Such models, often

\footnotetext{
a e-mail: henning.bahl@outlook.de (corresponding author)
}

motivated by theoretical arguments or observational puzzles (such as dark matter), are therefore equally viable in the light of the Higgs observation.

The MSSM [7-9] is one of the best studied models with an extended Higgs sector. In contrast to the case of the SM, the MSSM contains two Higgs doublet fields. This results in five physical Higgs bosons instead of the single Higgs boson in the SM. These are (in the $\mathcal{C P}$-conserving case, which is assumed throughout this paper) the light and heavy $\mathcal{C P}$-even Higgs bosons, $h$ and $H$, the $\mathcal{C} \mathcal{P}$-odd Higgs boson, $A$, and the charged Higgs bosons, $H^{ \pm}$. At tree-level the Higgs sector is determined by the ratio of the two vacuum expectation values, $\tan \beta$, and the mass of the $\mathcal{C P}$-odd Higgs boson, $M_{A}$. In addition, the MSSM predicts two scalar partners for all fermions as well as fermionic partners for all bosons, which for the electroweak gauge bosons and Higgs bosons are the so-called neutralinos and charginos, or electroweakinos.

In order to facilitate collider searches for the additional MSSM Higgs bosons a set of new benchmark scenarios for MSSM Higgs boson searches at the LHC has been proposed recently $[10,11]$. The scenarios are compatible - at least over wide portions of their parameter space - with the most recent LHC results for the properties of the Higgs boson at $125 \mathrm{GeV}$ [3-6] and the bounds on masses and cross sections of new SUSY particles, as well as with global fits of the phenomenological MSSM, see e.g. [12-14]. Each scenario contains one $\mathcal{C P}$-even scalar with a mass around $125 \mathrm{GeV}$ which is identified with the observed Higgs boson. Nevertheless, the scenarios differ significantly in their predictions for the light Higgs-boson phenomenology (within the allowed uncertainties), in particular in the non-decoupling regime, as well as in the phenomenology of the additional, so far undetected Higgs bosons. In many cases, such differences occur due to the presence of additional light particles like, for example, the above-mentioned electroweakinos. 
The search for the additional Higgs bosons will continue during LHC Run 3 and subsequently at the high luminosity (HL)-LHC [15]. At possible future $e^{+} e^{-}$colliders, such as the International Linear Collider (ILC), the Compact Linear Collider (CLIC), the Future Circular Collider (FCC)-ee or the Circular Electron Positron Collider (CEPC), the search for extended Higgs sectors can be performed either directly, via the search for new Higgs bosons, or indirectly, via the precise measurements of the properties of the Higgs boson at $125 \mathrm{GeV}$. The new benchmark scenarios, due to their distinct phenomenology of the MSSM Higgs sector, can be employed to assess the reach of current and future colliders.

In this paper we present the HL-LHC and ILC ${ }^{1}$ sensitivities to heavy Higgs bosons in the $M_{h}^{125}$ scenario [10], in which all supersymmetric particles are heavy, leaving essentially a Two-Higgs-Doublet Model (THDM)-like Higgs sector at the electroweak scale, and the $M_{h}^{125}(\tilde{\chi})$ scenario [10], which features light neutralinos and charginos. For the low $\tan \beta$ region we furthermore study the analogous scenarios $M_{h, \mathrm{EFT}}^{125}$ and $M_{h, \mathrm{EFT}}^{125}(\tilde{\chi})$, respectively, which employ a dedicated effective field theory (EFT) calculation and a varying SUSY mass scale [11]. In addition, we define in this work a new benchmark scenario in which the decays of the Higgs bosons to pairs of bottom quarks are enhanced. A selection of our results has already been summarized in the report of the HL/HE-LHC Higgs working group [15].

This paper is organized as follows: In Sect. 2 we briefly review the MSSM benchmark models used in this study and introduce the new benchmark scenario with enhanced Higgsbottom-quark couplings. In Sect. 3 we discuss the experimental input and the projections both for the direct and indirect searches at the HL-LHC and two ILC running scenarios. We present the exclusion reach of HL-LHC heavy Higgs boson searches and the indirect sensitivity from HL-LHC Higgs rate measurements in Sect. 4. In Sect. 5 we investigate constraints on BSM physics that can be obtained from the HL-LHC results in combination with precision Higgs rate measurements at the ILC. In this context we analyze both the case where the future rate measurements agree with the SM prediction and the case where the rates agree with the predictions of possible realizations of the MSSM Higgs sector in nature. We conclude in Sect. 6.

\section{MSSM benchmark models}

We perform our study of the physics potential of the HL-LHC and the ILC in the exploration of heavy neutral Higgs bosons in the framework of five MSSM benchmark scenarios. This

\footnotetext{
1 We focus on the ILC as specific realization of an $e^{+} e^{-}$Higgs factory since it is the currently most advanced project and anticipated precisions are based on full detector simulations.
}

allows us to compare the sensitivity of direct searches for heavy neutral Higgs bosons with the indirect sensitivity from precision measurements of the light Higgs boson signal rates. The benchmark scenarios chosen here feature quite different predictions for the considered search channels and for the rates of the Higgs boson at $125 \mathrm{GeV}$, thus providing a certain variety in the phenomenology of the two approaches and for the different collider scenarios.

Four of the benchmark scenarios chosen here were proposed in Refs. [10,11]. We shall furthermore define one new scenario in this work in which the sensitivity of searches for heavy Higgs boson decays to bottom quarks is enhanced (see below). All considered scenarios are defined as twodimensional planes in $M_{A}$ and $\tan \beta$, the two parameters that fix the MSSM Higgs-boson sector at tree-level. We emphasize that the five benchmark scenarios considered in this work feature the decoupling limit [16], i.e. the light $\mathcal{C} \mathcal{P}$-even Higgs boson acquires SM-like tree-level couplings if $M_{A}$ becomes large, $M_{A} \gg M_{Z}$. In scenarios where loop corrections to the Higgs-boson mass matrix lead to an accidental alignment without decoupling [16-20], lower bounds on $M_{A}$ from the measurements of the rates of the Higgs boson at $125 \mathrm{GeV}$ become much weaker $[10,19]$. While scenarios featuring at least one additional Higgs boson that is lighter than the one at $125 \mathrm{GeV}$ are tightly constrained within the MSSM [10] and not considered in the present paper, such a situation can occur generically in singlet extensions of the Higgs sector, see e.g. Refs. [21-28].

The theory predictions for the Higgs-boson masses, couplings and branching ratios are obtained from FeynHiggs (see below for more details on specific versions employed in this work) [29-38]. For Higgs production via gluon fusion we make use of SusHi (version 1.7.0) [39-51]. For Higgs production via bottom-quark annihilation "matched predictions" are employed [52-55].

Standard scenarios: $M_{h}^{125}$ and $M_{h}^{125}(\tilde{\chi})$

The $M_{h}^{125}$ scenario [10] is characterized by relatively heavy superparticles, such that the Higgs phenomenology at the LHC resembles that of a THDM with MSSM-inspired Higgs couplings. The light Higgs boson has SM-like couplings, and in the region $M_{A} \lesssim 1.9 \mathrm{TeV}$ the heavy Higgs bosons can only decay into SM particles (including the light SMlike Higgs boson). For larger values of $M_{A}$ decays into electroweakinos (EWinos) are kinematically open. In contrast, the $M_{h}^{125}(\tilde{\chi})$ scenario [10] features light EWinos via the choice of relatively small values of the bino, wino and Higgsino mass parameters, $M_{1}, M_{2}$ and $\mu$, respectively. This leads to sizable decay rates of the heavy Higgs bosons $H$ and $A$ into charginos and neutralinos throughout the parameter plane, thus diminishing the event yield of the $\tau^{+} \tau^{-}$and $b \bar{b}$ final state signatures that are used to search for the additional 
Higgs bosons at the LHC. Furthermore, the branching ratio of the light Higgs boson $h$ into a pair of photons is enhanced for small values of $\tan \beta$ due to loop corrections involving light charginos.

In these two scenarios all other SUSY masses are fixed around the $\mathrm{TeV}$ scale. Consequently, in both scenarios, the light Higgs-boson mass becomes too small (within experimental and theoretical uncertainties, as taken over from Ref. [10], where a theory uncertainty of $\pm 3 \mathrm{GeV}$ was assumed [31]), $M_{h} \lesssim 122 \mathrm{GeV}$, for $\tan \beta \lesssim 6$, rendering this parameter region phenomenologically inconsistent with the observed state at $125 \mathrm{GeV}$. For these two standard scenarios we used FeynHiggs version 2.14.3. More details and a discussion of the current constraints from Higgs-boson searches and rate measurements in these two scenarios can be found in Ref. [10].

Low-tan $\beta$ (EFT) scenarios: $M_{h, \mathrm{EFT}}^{125}$ and $M_{h, \mathrm{EFT}}^{125}(\tilde{\chi})$

The $M_{h, \mathrm{EFT}}^{125}$ and $M_{h, \mathrm{EFT}}^{125}(\tilde{\chi})$ scenarios [11] have similar phenomenological properties as the standard scenarios described above, but are in agreement with the observed Higgs-boson mass value at lower values of $\tan \beta$. A light Higgs-boson mass $M_{h} \simeq 125 \mathrm{GeV}$ is achieved by tuning the scalar top masses to very large values for every point in the parameter plane. Accordingly, for these scenarios we employ the THDM as effective field theory (EFT) below the SUSY scale to calculate the higher-order corrections to the Higgs-boson masses and self-energies [36]. The $M_{h \text {,EFT }}^{125}$ scenario resembles the $M_{h}^{125}$ scenario, while the $M_{h, \mathrm{EFT}}^{125}(\tilde{\chi})$ scenario is phenomenologically similar to the $M_{h}^{125}(\tilde{\chi})$ scenario, i.e. it features light electroweakinos. These scenarios are discussed in detail in Ref. [11]. For these two EFT scenarios we used an extended version of FeynHiggs 2.14.3, which includes the THDM as EFT below the SUSY scale [36]. This feature was officially released (alongside other changes) in version 2.16.0.

Large $H / A \rightarrow b \bar{b}$ scenario(s)

In the MSSM the relation between the experimentally measured bottom-quark mass, $m_{b}$, and the bottom-quark Yukawa coupling, $h_{b}$, is affected by loop corrections which are enhanced for large $\tan \beta$. These corrections are summarized in the parameter $\Delta_{b} \propto \mu \tan \beta$ [56-61] according to

$h_{b} \sim \frac{m_{b}}{1+\Delta_{b}}$.

Consequently, large negative values of $\mu$ enhance the bottomquark Yukawa coupling and thus lead to enhanced rates of Higgs-boson decays to bottom quarks as well as of Higgs boson production in association with a bottom-quark pair. This enhancement is particularly strong for the heavy non-
SM Higgs bosons $H$ and $A$, while the SM-like light Higgs boson $h$ is only affected to a much lesser extent, as these corrections are suppressed in the decoupling limit. ${ }^{2}$ The Higgsino mass parameter $\mu$ can therefore via $\Delta_{b}$ lead to a change in the relative strengths of the Higgs boson couplings to bottom quarks and to $\tau$-leptons. This, in turn, changes the relative sensitivity of heavy Higgs boson searches in $b \bar{b}$ and $\tau^{+} \tau^{-}$final states.

In order to investigate this change in sensitivity we define one new benchmark scenario, which we denote by $M_{h}^{125}(\mu=$ $-2 \mathrm{TeV})$ or the short-hand notation $M_{h}^{125, \mu-3}$. The SUSY input parameters are chosen to be

$$
\begin{aligned}
& M_{Q_{3}}=M_{U_{3}}=M_{D_{3}}=1.5 \mathrm{TeV}, \quad M_{L_{3}}=M_{E_{3}}=2 \mathrm{TeV}, \\
& \mu=-2 \mathrm{TeV}, \\
& M_{1}=1 \mathrm{TeV}, \quad M_{2}=1 \mathrm{TeV}, M_{3}=2.5 \mathrm{TeV}, \\
& X_{t}=2.8 \mathrm{TeV}, \quad A_{b}=A_{\tau}=A_{t} .
\end{aligned}
$$

All parameters are defined in the on-shell scheme. For the SM input parameters, we follow the recommendations of the LHC-HXSWG [63].

Apart from $\mu$, all parameters are chosen as in the $M_{h}^{125}$ scenario, in which $\mu=+1 \mathrm{TeV}$. In addition to this default "large $H / A \rightarrow b \bar{b}$ scenario", we also investigate two other choices of $\mu(\mu=-1 \mathrm{TeV}$ and $\mu=-3 \mathrm{TeV})$. For the scenarios with negative $\mu$ we employ FeynHiggs version 2.14.4 which includes additional two-loop $\Delta_{b}$ corrections [64-66] in the Higgs-boson decays.

In Fig. 1(left) we show the current LHC constraints at 95\% confidence level (CL) from heavy Higgs searches in the $b \bar{b}$ and $\tau^{+} \tau^{-}$final states as well as from Higgs signal rate measurements for the standard $M_{h}^{125}$ scenario (black lines and very-light-gray shading) and the three variants of the $M_{h}^{125}$ scenario with different choices of the $\mu$ parameter: $\mu=-1 \mathrm{TeV}$ (blue lines and light-gray shading), $\mu=-2 \mathrm{TeV}$ (i.e. $M_{h}^{125, \mu-}$, green line and medium-gray shading), $\mu=-3 \mathrm{TeV}$ (red line and dark-gray shading). The heavy Higgs boson search limits are derived from the ATLAS $p p \rightarrow H / A \rightarrow b \bar{b}$ search [67] with $27.8 \mathrm{fb}^{-1}$ of data, and from the CMS $p p \rightarrow H / A \rightarrow \tau^{+} \tau^{-}$search [68] with $35.9 \mathrm{fb}^{-1}$ of data, both at a center-of-mass energy of $13 \mathrm{TeV}$, using HiggsBounds [69-74]. The indirect constraints from Higgs rate measurements are evaluated with HiggsSignals (version 2 .3 .0) [75,76] by means of a negative log-likelihood ratio (LLR) test with the SM as alternative hypothesis, and approximating the likelihood with a $\chi^{2}$ function. This test uses Run-1 [3] and recent Run-2 results

\footnotetext{
${ }^{2}$ Corresponding $\Delta_{\tau}$ effects are substantially smaller and not taken into account in a resummed way.

3 This is similar to the change in the $\mu$ values in the previously proposed $m_{h}^{\text {mod }+}$ scenario [62], where $\mu= \pm 200, \pm 500, \pm 1000 \mathrm{GeV}$ had been chosen.
} 


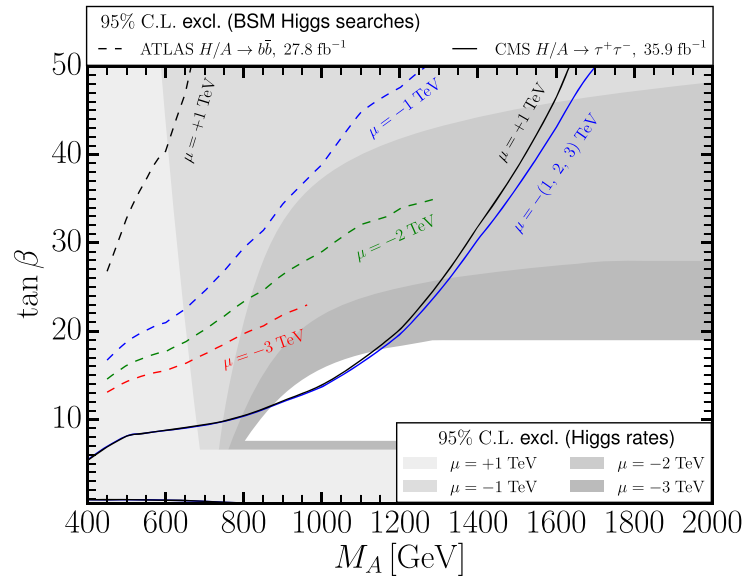

Fig. $1 M_{h}^{125}$ scenario in comparison with the proposed new $M_{h}^{125}(\mu=$ $-2 \mathrm{TeV}$ ) scenario and the other choices of $\mu=-1 \mathrm{TeV},-3 \mathrm{TeV}$, shown in the $\left(M_{A}, \tan \beta\right)$ parameter plane. Left panel: Current experimental constraints from heavy Higgs searches in the $b \bar{b}$ [67] (dashed lines) and $\tau^{+} \tau^{-}$[68] (solid lines) final state and $125 \mathrm{GeV}$ Higgs rate

up to around $80 \mathrm{fb}^{-1}$ from ATLAS [77] and CMS [78-87]. Figure 1(left) clearly illustrates that $p p \rightarrow H / A \rightarrow b \bar{b}$ searches become more sensitive for scenarios with large negative $\mu$ values due to the enhancement of the bottom-quark Yukawa coupling, as the excluded regions probe lower values of $\tan \beta$ for larger negative $\mu$ values. ${ }^{4}$ It is noteworthy that the exclusion limit from $p p \rightarrow H / A \rightarrow \tau^{+} \tau^{-}$searches does not vary significantly with $\mu .^{5}$ This is because the $p p \rightarrow H / A \rightarrow b \bar{b}$ signal rate profits from an enhancement in the production (in the $g g \rightarrow b \bar{b} H / A$ production mode) and in the decay branching ratio (BR) of the $H / A \rightarrow b \bar{b}$ decay, while the $p p \rightarrow H / A \rightarrow \tau^{+} \tau^{-}$signal rate only gains from the enhancement in the production rate whereas in combination with the decay rate $\mathrm{BR}\left(H / A \rightarrow \tau^{+} \tau^{-}\right)$a large compensation of $\Delta_{b}$ effects occurs [88]. Still, we observe that heavy Higgs-boson searches in the $b \bar{b}$ final state cover significantly less parameter space in the benchmark plane than searches in the $\tau^{+} \tau^{-}$final state, regardless of the choice of $\mu$.

We can furthermore see in Fig. 1(left) that, currently, the indirect exclusion derived from $125 \mathrm{GeV}$ Higgs signal rate measurements is stronger than the $H / A \rightarrow b \bar{b}$ (but not $H / A \rightarrow \tau^{+} \tau^{-}$) search limits for all choices of $\mu$. At larger $\tan \beta$ values these indirect constraints dominantly originate from deviations of the light Higgs-boson

\footnotetext{
${ }^{4}$ The exclusion lines for $\mu=-2 \mathrm{TeV}$ and $-3 \mathrm{TeV}$ terminate, as for larger $\tan \beta$ values the light Higgs boson mass quickly decreases (see also the right panel of Fig. 1) and the prediction of Higgs-boson masses is affected by large uncertainties.

5 The exclusion contours derived from the CMS $p p \rightarrow H / A \rightarrow \tau^{+} \tau^{-}$ search are practically identical for the choices $\mu=-1,-2$ and $-3 \mathrm{TeV}$, and therefore plotted as a single contour.
}

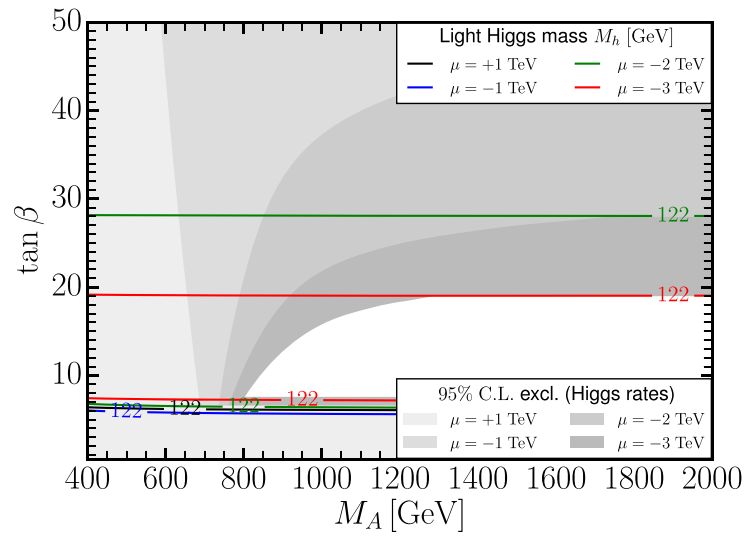

measurements (gray filled regions); right panel: contours for the lowest acceptable value of the light-Higgs boson mass, $M_{h}=122 \mathrm{GeV}$ (taking into account a theory uncertainty of $\pm 3 \mathrm{GeV}$ ), with the Higgs rate constraints (from the left panel) superimposed

Yukawa coupling to bottom quarks. By approaching the decoupling limit, i.e. with increasing $M_{A}$, the allowed parameter space opens towards larger $\tan \beta$ values. However, at around $\left(M_{A}, \tan \beta\right) \sim(1.75 \mathrm{TeV}, 28)$ for $\mu=-2 \mathrm{TeV}$, and at around $\left(M_{A}, \tan \beta\right) \sim(1.3 \mathrm{TeV}, 19)$ for $\mu=-3 \mathrm{TeV}$, and extending to larger $M_{A}$ values, the exclusion from the $125 \mathrm{GeV}$ Higgs measurements becomes a constant upper limit on $\tan \beta$ in this scenario. This is caused by the light Higgs-boson mass dropping below $122 \mathrm{GeV}$ (see the discussion above) for larger values of $\tan \beta$ for these two choices of $\mu$. Similarly, the light Higgs-boson mass drops below $122 \mathrm{GeV}$ for $\tan \beta \leq 7$. This is illustrated in Fig. 1(right), which shows contours for the lowest acceptable value (taking into account the theoretical uncertainty) of the light-Higgs boson mass, $M_{h}=122 \mathrm{GeV}$, for the different choices of $\mu$.

\section{Experimental input for HL-LHC and ILC projections}

We assess the reach of direct LHC searches in the $\tau^{+} \tau^{-}$final state by applying the model-independent $95 \%$ CL limit projections for $6 \mathrm{ab}^{-1}$ by the CMS experiment $[15,89]$, serving as a proxy for a future ATLAS and CMS search combination using the full HL-LHC data. We implemented these limits - presented as one-dimensional (marginalized) cross section limits on either the gluon fusion or $b \bar{b}$-associated production mode - into HiggsBounds [69-74] to obtain the projected 95\% CL exclusion in our scenarios.

For LHC searches for heavy Higgs bosons decaying into $b \bar{b}$ or di-Higgs $(h h)$ final states, unfortunately, no experimental HL-LHC projection has been performed by ATLAS 
and CMS. ${ }^{6}$ In order to estimate the future sensitivity of these searches, we approximate the future $95 \% \mathrm{CL}$ limit, $\sigma_{\mathrm{HL}-\mathrm{LHC}}^{95 \%}$, by rescaling the current expected limit, $\sigma_{\text {current }}^{95 \% \mathrm{CL}}$, based on the current integrated luminosity, $\mathcal{L}_{\text {current }}$, at $\sqrt{s}=13 \mathrm{TeV}$, by the expected increase of statistics to the future combined ATLAS and CMS integrated luminosity, $\mathcal{L}_{\mathrm{HL}-\mathrm{LHC}}=6 \mathrm{ab}^{-1}$, at a slightly higher center-of-mass energy of $14 \mathrm{TeV},{ }^{7}$

$\sigma_{\mathrm{HL}-\mathrm{LHC}}^{95 \% \mathrm{CL}}=\sigma_{\text {current }}^{95 \% \mathrm{CL}} \cdot \sqrt{\frac{\mathcal{L}_{\text {current }}}{\mathcal{L}_{\mathrm{HL}-\mathrm{LHC}}}}$.

Obviously, this estimate has to be taken with a grain of salt, as we assumed the full uncertainty to improve like a statistical uncertainty. Our projection of heavy Higgs boson searches in the $b \bar{b}$ final state is based on the current ATLAS search with $\mathcal{L}_{\text {current }}=28.7 \mathrm{fb}^{-1}$ [67], while we use the current CMS $H \rightarrow h$ analysis [91] based on $\mathcal{L}_{\text {current }}=36 \mathrm{fb}^{-1}$ (which combined several final states) for the projection of searches in the di-Higgs final state. The current limit of these searches is strongly dominated by statistical uncertainties, which motivates our naive projection approach. Only more sophisticated projection studies by ATLAS and/or CMS would give a more reliable picture.

We do not take into account direct searches in the $A \rightarrow Z h$ final states, as in the MSSM they are expected to be less relevant than the $H \rightarrow h h$ searches [11]. We also do not assess the HL-LHC reach of searches for heavy Higgs bosons in the $t \bar{t}$ final state, as there are no official projections available and since the limit is strongly influenced by interference effects between signal and the SM background, as discussed in Refs. [92-98], and is therefore rather model-dependent. It is clear that these searches will be able to constrain low values of $\tan \beta[99,100]$, where the heavy Higgs bosons predominantly decay into di-top final states. Thus, in particular in the EFT-scenarios they will have a significant impact at low values of $\tan \beta$, see for comparison Fig. 6 in Ref. [100] for the current hMSSM [101-105] limits obtained with an integrated luminosity of $36 \mathrm{fb}^{-1}$. Similarly, also for the charged Higgs-boson searches at the HL-LHC no official projections

\footnotetext{
6 See Ref. [90] for a theorists' analysis of the HL-LHC prospects of $H \rightarrow h h$ searches for various final states.

7 In our naive projection of the heavy Higgs-boson searches in $b \bar{b}$ and $h h$ final states we neglect effects related to the slightly higher center-ofmass energy $\sqrt{s}=14 \mathrm{TeV}$ in the HL-LHC runs. As the cross sections for the background processes increase with respect to the current run, the resulting upper cross section limit at $14 \mathrm{TeV}$ will be larger than our estimated $\sigma_{\mathrm{HL}-\mathrm{LHC}}^{95 \% \mathrm{CL}}$. This degradation however depends strongly on the experimental background estimation methods and the specific process. Furthermore, it will most likely be overcome by the simultaneous increase in the signal cross section for $14 \mathrm{TeV}$, thus leading overall to a net gain in sensitivity in particular at large Higgs boson masses. In that sense, our projection may be regarded as a conservative estimate.
}

in benchmark planes are available. ${ }^{8}$ Consequently, we also leave charged Higgs sensitivity studies for future work.

We estimate the indirect reach through Higgs rate measurements by using the detailed HL-LHC signal strength projections for the individual Higgs production times decay modes, including the corresponding correlation matrix, as evaluated by the ATLAS and CMS collaborations (see Tab. 35 in Ref. [15]). These projections assume the evolution of systematic uncertainties as estimated by the HL-LHC Working Group 2 (see Section 1.1.3 in Ref. [15] and references therein for details) and are referred to by future scenario S2 or 'YR18' systematic uncertainties. We furthermore take cross-correlations of theoretical rate uncertainties between future ATLAS and CMS measurements into account, assuming that theoretical and parametric uncertainties are halved with respect to current estimates [63] (as prescribed in S2). All projections of Higgs-boson rate measurements are implemented into HiggsSignals, which we use to evaluate the projected reach in the MSSM parameter space.

For the indirect reach of Higgs signal rate measurements at the ILC we consider two future scenarios: First, the initial stage scenario at $\sqrt{s}=250 \mathrm{GeV}$ with $2 \mathrm{ab}^{-1}$ of data (denoted ILC250), and second, the ILC program including a second run at $350 \mathrm{GeV}$ with $0.2 \mathrm{ab}^{-1}$ of data, and a third run at $\sqrt{s}=500 \mathrm{GeV}$ with $4 \mathrm{ab}^{-1}$ of data (for brevity we denote this future scenario by ILC500). All ILC runs assume $-80 \%$ polarization of electrons and $+30 \%$ polarization of positrons. The projected precisions of various Higgs-boson signal channels are listed in Tab. 21 and 22 of Ref. [107]. We implemented these future anticipated measurements in HiggsSignals. For the Higgs branching ratios, we assume that theoretical and parametric uncertainties are halved (as done for the HL-LHC, see above), while we assume a theoretical uncertainty on the $e^{+} e^{-} \rightarrow Z h$ $(\nu \bar{\nu} h)$ cross section of $0.5 \%$ (1\%) [108]. The projections for both future scenarios ILC250 and ILC500 are combined in our analysis with the projected HL-LHC Higgs rate measurements.

For illustration, we shall furthermore compare for specific MSSM parameter points the predictions of Higgs coupling scale factors [63], $\kappa_{i}$, with the anticipated precision of the future $\kappa$ determination at the HL-LHC and ILC, taken from Tabs. 4 and 5 of Ref. [107]. Note that these projections of the future $\kappa_{i}$ determination are based on identical experimental input as in our study, but have been evaluated in a Bayesian statistical analysis instead of a log-likelihood ratio (LLR) test as employed here.

\footnotetext{
${ }^{8}$ In Refs. [15,106] the sensitivity of charged Higgs boson searches was estimated for a set of minimal supergravity (mSUGRA) benchmark points. This model-dependent study indicates that the charged Higgsboson search is less sensitive than neutral Higgs-boson searches.
} 


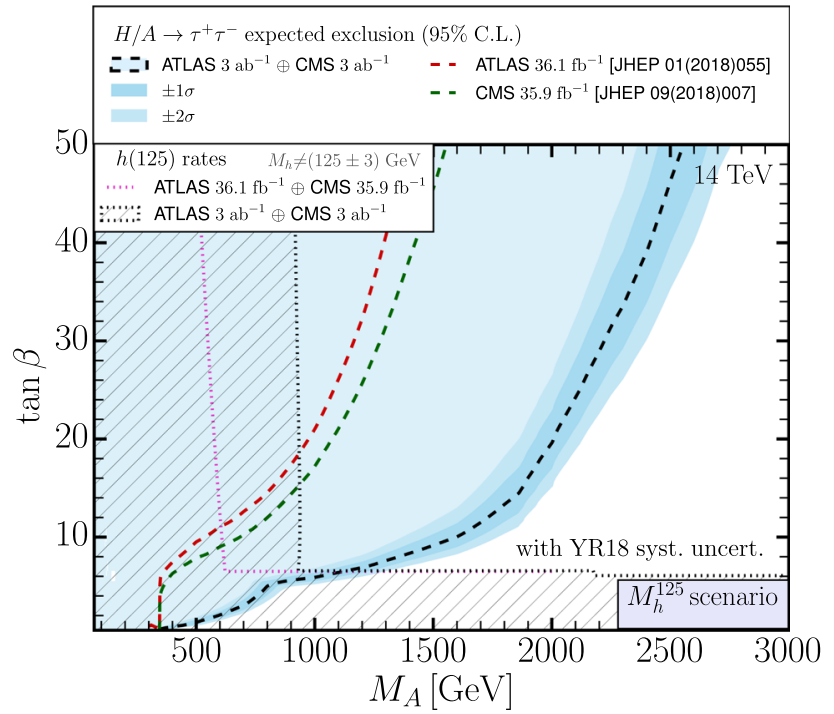

Fig. 2 HL-LHC projections in the $M_{h}^{125}$ scenario, assuming YR18 systematic uncertainties (scenario S2 in Ref. [15]). The dashed black curve and blue filled region indicate the expected HL-LHC reach via direct heavy Higgs searches in the $\tau^{+} \tau^{-}$channel with $6 \mathrm{ab}^{-1}$ of data (with the dark blue regions indicating the 1 and $2 \sigma$ uncertainty), whereas the red and green dashed lines show the expected limit from current searches in this channel by ATLAS [109] and CMS [68], respectively. The current and future HL-LHC sensitivity via combined ATLAS and CMS Higgs rate measurements is shown as magenta and black dotted contours, respectively (the latter being accompanied with a hatching of the prospectively excluded region)

\section{Sensitivity of the HL-LHC}

We start with a discussion of the future HL-LHC sensitivity via rate measurements and direct searches for heavy MSSM Higgs bosons. In the subsequent figures we present in the $\left(M_{A}, \tan \beta\right)$ planes of the considered MSSM benchmark scenarios the projected direct and indirect 95\% CL sensitivity of a future combination of ATLAS and CMS data at the HLLHC. For comparison, we also include the current sensitivity of the corresponding searches and measurements, as presented in part in Refs. $[10,11]$. In the next section we shall address the improvements of the indirect reach obtained by ILC measurements.

Our projections in the $M_{h}^{125}$ scenario are presented in Fig. 2. Corresponding planes for the $M_{h}^{125}(\tilde{\chi})$ and $M_{h \text {.EFT }}^{125}$ scenario are given in Figs. 3 and 4, respectively. In the figures we show the current limit (magenta dotted line) for the indirect reach of the LHC in the benchmark scenarios, as evaluated in Refs. [10,11], as well as the expected limit from current direct BSM Higgs searches by ATLAS [109] (red dashed line) ${ }^{9}$ and CMS [68] (green dashed line) in the $\tau^{+} \tau^{-}$ final state, using $\sim 36 \mathrm{fb}^{-1}$ of data from Run 2 at $13 \mathrm{TeV}$.

\footnotetext{
9 Very recently the corresponding result based on $139 \mathrm{fb}^{-1}$ has become available [110]. Including this would not affect our conclusions.
}

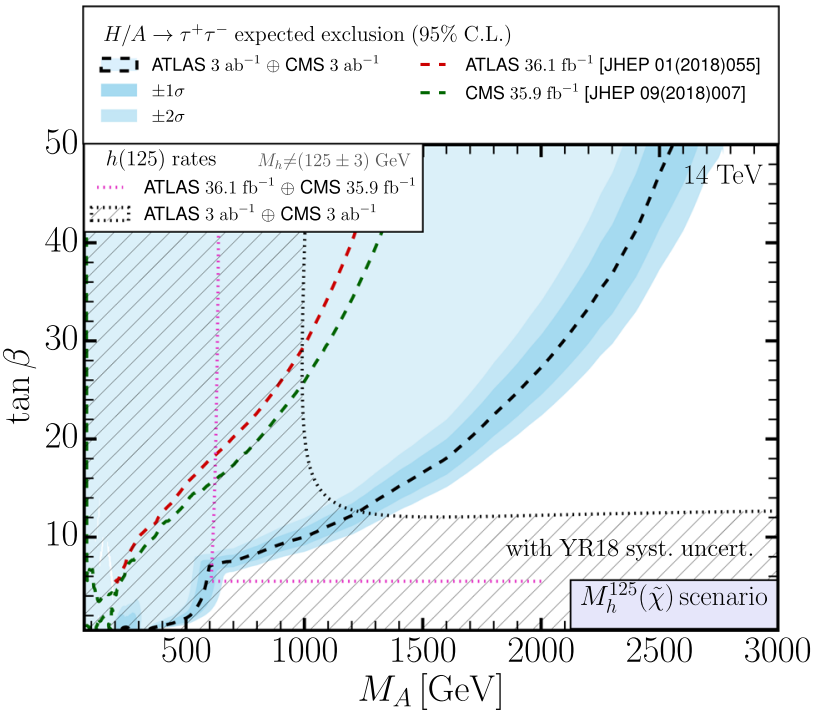

Fig. 3 HL-LHC projections in the $M_{h}^{125}(\tilde{\chi})$ scenario with the same color coding as in Fig. 2

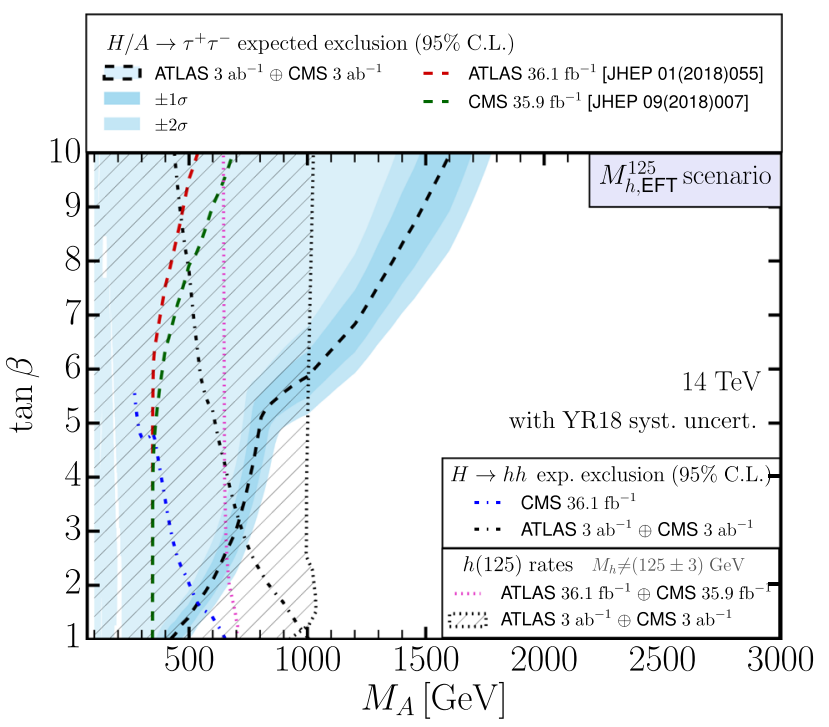

Fig. 4 HL-LHC projections in the $M_{h \text {,EFT }}^{125}$ scenario with the same color coding as in Fig. 2. The blue and black dash-dotted lines show the current CMS [91] and future HL-LHC expected 95\% CL limit from a combination of $H \rightarrow h h$ searches (see Sect. 3 for details)

The dashed black curve and blue filled region indicate the HL-LHC reach via direct heavy Higgs searches in the $\tau^{+} \tau^{-}$ channel with $6 \mathrm{ab}^{-1}$ of data (with the dark blue regions indicating the 1 and $2 \sigma$ experimental uncertainty). The future HL-LHC sensitivity via combined ATLAS and CMS Higgs rate measurements is shown as black dotted contours, accompanied with a hatching of the prospectively excluded region.

Within the $M_{h}^{125}$ scenario, displayed in Fig. 2, the indirect constraints at the HL-LHC have the sensitivity for a prospective exclusion limit that is given by a nearly vertical band extending to $M_{A}$ values of around $900 \mathrm{GeV}$ and 
by a nearly horizontal band with $\tan \beta$ values of up to 6 . The nearly vertical band arises from the measurements of the Higgs signal strengths, while the nearly horizontal band is due to the prediction for the mass of the SM-like Higgs boson. In the nearly horizontal band this prediction is below $122 \mathrm{GeV}$ for the parameters of the $M_{h}^{125}$ scenario, such that the interpretation of the observed Higgs signal in terms of the light $\mathcal{C} \mathcal{P}$-even MSSM Higgs boson $h$ is incompatible in this specific benchmark scenario with the experimental mass value for the adopted theory uncertainty of $3 \mathrm{GeV}$. Figure 2 shows that in this scenario the indirect sensitivity of the Higgs rate measurements at the HL-LHC is not sufficient to probe parameter regions that are not covered by the direct Higgs searches (black dashed line). Those direct searches in the $\tau^{+} \tau^{-}$final state will probe the parameter space up to $M_{A} \lesssim 2.5 \mathrm{TeV}$ for the highest displayed $\tan \beta$ values of $\tan \beta \sim 50$. At $\tan \beta=20$ the reach extends up to $M_{A} \lesssim 2000 \mathrm{GeV}$. The change in the curvature of the black dashed line around $M_{A} \sim 1.9 \mathrm{TeV}$ can be understood from the fact that for larger values of $M_{A}$ decays of $H$ and $A$ into electroweakinos open, thus diminishing the event yield of the $\tau^{+} \tau^{-}$final state. The kink in the exclusion boundary at $M_{A} \sim 800 \mathrm{GeV}$ is caused by a transition of the main production channel from gluon fusion (low $\tan \beta$ values) to bottom quark associated production (high $\tan \beta$ values). ${ }^{10}$ In this scenario the prospective combined sensitivity from direct and indirect searches in the absence of a signal would yield a lower bound on $M_{A}$ of about $M_{A} \gtrsim 1200 \mathrm{GeV}$. In order to correctly interpret this result, the following should be taken into account. As explained above, this bound is not a consequence of prospective Higgs signal strength measurements at the HL-LHC, but it is rather driven by the direct Higgs search reach in combination with the Higgs-mass prediction. Since by definition for this benchmark scenario all parameters except $M_{A}$ and $\tan \beta$ are set to fixed values, the adopted theoretical uncertainty of the Higgs-mass prediction has a major impact on the resulting bound. For a smaller theoretical uncertainty the allowed region in this scenario would be shifted to larger $\tan \beta$ values, so that the lower bound on $M_{A}$ would rise to values above $2 \mathrm{TeV}$. On the other hand, in scenarios where the prediction for the mass of the light Higgs boson is compatible with the measured Higgs-boson mass also for low $\tan \beta$ values, the indirect constraints on $M_{A}$ from the rate measurements can exceed the sensitivity from the direct searches (see the discussion below).

The picture is somewhat different in the $M_{h}^{125}(\tilde{\chi})$ scenario. Here the large branching ratio of the heavy neutral Higgs boson decaying to charginos and neutralinos already at lower values of $M_{A}$ leads to a strongly reduced direct reach of

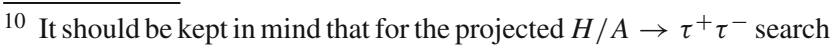
sensitivity we used the one-dimensional profiled cross section limits for the two relevant production modes.
}

$H / A \rightarrow \tau^{+} \tau^{-}$searches. The kink in the exclusion boundary at $M_{A} \sim 600 \mathrm{GeV}$ is as in Fig. 2 caused by a transition of the most sensitive production channel from gluon fusion (at low $\tan \beta$ values) to bottom quark associated production (at high $\tan \beta$ values). At $\tan \beta=20$ the reach in the $M_{h}^{125}(\tilde{\chi})$ scenario is significantly reduced to $M_{A} \lesssim 1700 \mathrm{GeV}$ compared to the $M_{h}^{125}$ scenario with $M_{A} \lesssim 2000 \mathrm{GeV}$. On the other hand, at large values of $\tan \beta \sim 50$ and thus large $M_{A}$ the reach is only slightly weaker than in the $M_{h}^{125}$ scenario, as for those $M_{A}$ values in both scenarios decays into electroweakinos are kinematically open. In order to further strengthen the impact of direct searches it would be useful to supplement the searches in the $\tau^{+} \tau^{-}$and $b \bar{b}$ final states with dedicated searches for the decays of $H$ and $A$ to charginos, neutralinos and in general also to sleptons (see Refs. [111-127] for related experimental and phenomenological studies).

In this scenario the Higgs rate measurements are an important complementary probe. In the $M_{h}^{125}(\tilde{\chi})$ scenario the Higgs rate measurements at the HL-LHC have the sensitivity to exclude values of $M_{A} \leq 950 \mathrm{GeV}$ and $\tan \beta \leq 12.5$. The bound on $M_{A}$ arises in a similar way as for the $M_{h}^{125}$ scenario discussed above from the slight deviations in the Higgs couplings compared to the SM values that are caused by $M_{A}$ values below the asymptotic region of decoupling. The displayed bound on $\tan \beta$, on the other hand, is not related to the Higgs-mass prediction as in Fig. 2 but is a consequence of the loop contribution of light charginos to the $h \rightarrow \gamma \gamma$ partial width. For values of $\tan \beta \leq 12.5$ the enhancement of the $h \rightarrow \gamma \gamma$ partial width is so large that the HL-LHC has the potential to exclude this parameter region via the precise measurements of the $h \rightarrow \gamma \gamma$ rates, which have an anticipated precision of $2.6 \%$ [15]. The combination of direct and indirect bounds yields a prospective lower limit of $M_{A} \gtrsim 1250 \mathrm{GeV}$ in the $M_{h}^{125}(\tilde{\chi})$ scenario. This bound is less sensitive to the theoretical uncertainties of the Higgs-mass prediction than for the $M_{h}^{125}$ scenario but instead depends on the mass scale of the charginos that has been chosen in the $M_{h}^{125}(\tilde{\chi})$ scenario.

In fact, the ongoing searches for electroweakinos in the mass-compressed region put the $M_{h}^{125}(\tilde{\chi})$ scenario under some tension as the parameters of the electroweakino sector are fixed to rather low values of $M_{1}=160 \mathrm{GeV}$ and $M_{2}=\mu=180 \mathrm{GeV}$. However, one can increase the mass parameters of the electroweakino sector by, for instance, $+100 \mathrm{GeV}$ without a major impact on the sensitivity reach of the $\tau^{+} \tau^{-}$searches. This can be understood from the fact that the heavy Higgs bosons, as long as the decay modes are kinematically open, still show dominant branching ratios into electroweakinos at low and moderate values of $\tan \beta$. On the other hand, increasing the electroweakino masses significantly lowers the effect of the chargino loop contributions on 


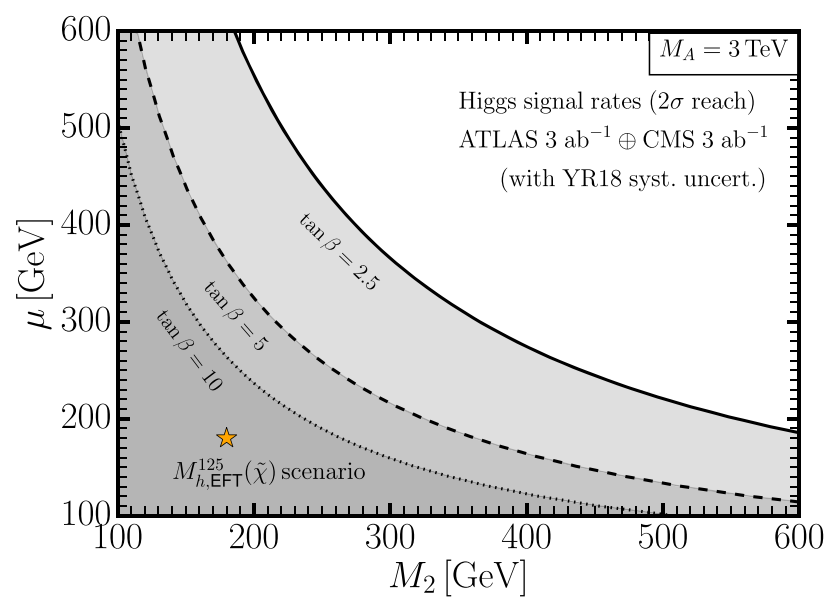

Fig. 5 Projected reach of future Higgs signal rate measurements at ATLAS and CMS with $3 \mathrm{ab}^{-1}$ assuming YR18 systematic uncertainties in the $\left(M_{2}, \mu\right)$ parameter plane around the $M_{h, \mathrm{EFT}}^{125}(\tilde{\chi})$ scenario (denoted by the orange star), for fixed $M_{A}=3 \mathrm{TeV}$. The solid, dashed and dotted contour lines and the corresponding gray areas indicate the $2 \sigma$ reach for $\tan \beta$ values of $2.5,5$ and 10 , respectively

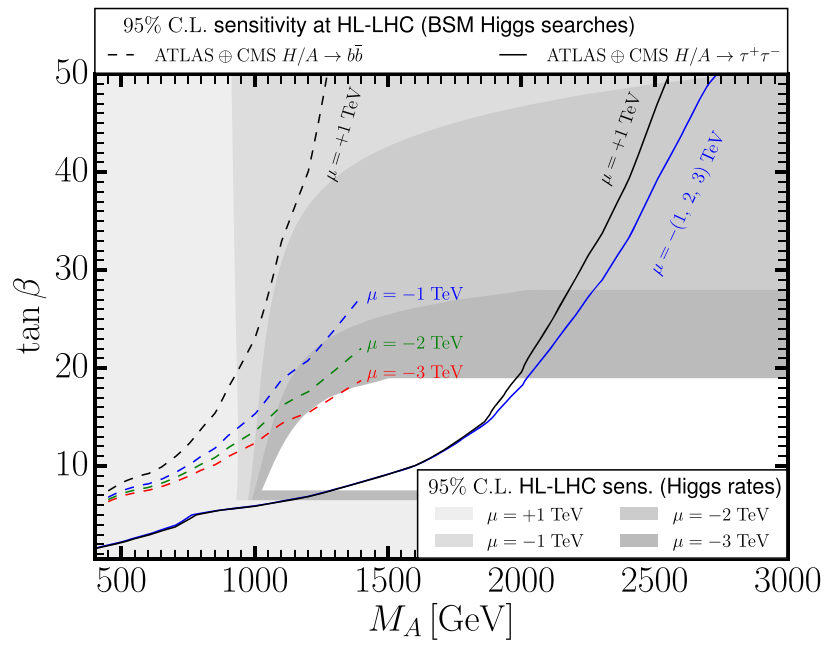

Fig. $6 \mathrm{HL}-\mathrm{LHC}$ projections for the proposed new $M_{h}^{125}(\mu=-2 \mathrm{TeV})$ scenario in comparison with the $M_{h}^{125}$ scenario and the other choices of $\mu=-1 \mathrm{TeV},-3 \mathrm{TeV}$, shown in the $\left(M_{A}, \tan \beta\right)$ parameter plane; the dashed and solid lines show the expected exclusion from heavy Higgs boson searches in the $b \bar{b}$ and $\tau^{+} \tau^{-}$final state, respectively, and the gray filled regions indicate the indirect reach of HL-LHC Higgs rate measurements

the $h \rightarrow \gamma \gamma$ partial decay width. ${ }^{11}$ We will investigate this effect in more detail below in the context of the $M_{h, \mathrm{EFT}}^{125}(\tilde{\chi})$ scenario (see Fig. 5).

\footnotetext{
${ }^{11}$ For even lighter electroweakinos than in this scenario, with masses below $M_{h} / 2$, the possibility of invisible decays of the observed Higgs boson $h$ arises. This decay leads to additional modifications of the Higgs rates and can also be searched for directly (see Sec. 6 of Ref. [15] for the HL-LHC prospects, and Refs. [120,128-131] for recent studies of SUSY models in the context of dark matter).
}

The $M_{h \text {.EFT }}^{125}$ scenario in Fig. 4 is only shown up to $\tan \beta=$ 10 , as this reflects the main feature of this scenario, which is to provide access to the low $\tan \beta$ region. Since this scenario allows a light Higgs-boson mass of $125 \mathrm{GeV}$ even for $\tan \beta$ values as low as $\tan \beta \sim 1$, the indirect reach through Higgs rate measurements is now almost vertical. The horizontal band yielding a lower bound on $\tan \beta$ in the $M_{h}^{125}$ scenario from the compatibility of the Higgs-boson mass prediction with $125 \mathrm{GeV}$ is not present in the $M_{h, \mathrm{EFT}}^{125}$ scenario. Since the $M_{h, \mathrm{EFT}}^{125}$ scenario does not feature light charginos, also the lower bound on $\tan \beta$ arising from precise measurements of the $h \rightarrow \gamma \gamma$ rates in the $M_{h}^{125}(\tilde{\chi})$ scenario does not occur in Fig. 4.

Again the indirect reach in $M_{A}$ is similar to the scenarios discussed beforehand, driven by the behavior of the couplings of the light $\mathcal{C P}$-even Higgs boson when approaching the decoupling limit, which is mostly a function of $M_{A}$ and hardly dependent on $\tan \beta$. The projected sensitivity at the HL-LHC in this scenario therefore corresponds to an almost vertical expected exclusion region for $\mathcal{C} \mathcal{P}$-odd Higgs boson masses $M_{A} \lesssim 1000 \mathrm{GeV}$. The direct search reach for heavy neutral Higgs bosons is similar to the $M_{h}^{125}$ scenario for the displayed $\tan \beta$ region. However, due to the fact that the low $\tan \beta$ region is not excluded by the light Higgs-boson mass predictions, the decay $H \rightarrow h h$ can cover some additional parameter space, up to $M_{A} \sim 1000 \mathrm{GeV}$ for $\tan \beta=1$. At low $\tan \beta$ searches for heavy neutral Higgs bosons in the di-top final state would be of relevance as well, but are not further discussed here (see Sect. 3). As a result, in the $M_{h \text { EFT }}^{125}$ scenario we find that the indirect sensitivity from the Higgs rate measurements has the largest coverage for $\tan \beta$ values up to $\tan \beta \sim 5.5$, while for higher values of $\tan \beta$ the direct searches for heavy Higgs bosons in the $\tau^{+} \tau^{-}$final state have the best prospects.

In order to cover the low-tan $\beta$ region, further experimental sensitivity studies for direct searches for $H / A \rightarrow t \bar{t}$, $H \rightarrow h h$ and $A \rightarrow Z h$ decays as well as heavy Higgs boson decays into electroweakinos are of interest (see Refs. [90,98] for recent theorists' projections of $H / A \rightarrow t \bar{t}$ and $H \rightarrow h h$, and Ref. [15] for experimental projections in different scenarios). The searches for decays to electroweakinos are of particular importance in both the $M_{h}^{125}(\tilde{\chi})$ and the $M_{h, \mathrm{EFT}}^{125}(\tilde{\chi})$ scenario, see also Refs. [10,11,123].

We now turn to the second EFT scenario, $M_{h, \mathrm{EFT}}^{125}(\tilde{\chi})$, with a light EWino spectrum. As for the case of the $M_{h}^{125}(\tilde{\chi})$ scenario discussed above, the HL-LHC measurement of the diphoton Higgs-boson signal rate has the potential to set a lower bound on $\tan \beta$ for the chosen values of the chargino masses. In fact, restricting ourselves to the $\tan \beta$ range between 1 and 10 that was originally proposed for this scenario, the entire $\left(M_{A}, \tan \beta\right)$ plane of the $M_{h, \mathrm{EFT}}^{125}(\tilde{\chi})$ scenario can be probed by the HL-LHC measurement of the di-photon Higgs- 
boson signal rate. Accordingly, this parameter plane could be excluded at the HL-LHC if no deviation from the SM prediction is observed. Therefore, instead of displaying the $\left(M_{A}\right.$, $\tan \beta$ ) plane, we instead investigate the reach of the HL-LHC in the $\left(M_{2}, \mu\right)$ parameter plane, where $M_{2}$ is the soft-breaking wino mass parameter and $\mu$ the Higgs mixing parameter. This is shown in Fig. 5, where we highlight the prospective $2 \sigma$ excluded region, assuming HL-LHC Higgs signal rate measurements that agree with the SM expectation. The results are shown for three different values of $\tan \beta=2.5,5,10$ and fixed $M_{A}=3 \mathrm{TeV}$. As can be seen in Fig. 5 , the reach in the chargino mass parameters $M_{2}$ and $\mu$ increases with decreasing $\tan \beta$, caused by a larger mixing of the charginos with decreasing $\tan \beta$, which directly impacts the $h \rightarrow \gamma \gamma$ partial decay width. Similarly, the largest values of the light chargino mass, $M_{\tilde{\chi}_{1}^{ \pm}}$, can be probed if $M_{2} \approx \mu$, as in this case the chargino mixing is large, and in turn, the Higgs boson coupling to charginos is maximized. For instance, for $\tan \beta=2.5(5)$ and $M_{2} \approx \mu$, light chargino masses up to $\sim 255$ (190) $\mathrm{GeV}$ can be probed at the $2 \sigma$ level (in this case, the heavier chargino mass is $\sim 410(320) \mathrm{GeV})$. In contrast, in case of a larger hierarchy, $M_{2} \gg \mu$ or $M_{2} \ll \mu$, the smaller of the two mass parameters has to be rather low in order to be able to probe the electroweakino sector via the di-photon signal strength measurements. The nominal values of $M_{2}$ and $\mu$ that were chosen in the definition of the $M_{h, \mathrm{EFT}}^{125}(\tilde{\chi})$ scenario, marked by an orange star in Fig. 5 , could be probed for $\tan \beta \lesssim 12.5$, which is in agreement with the findings in the $M_{h}^{125}(\tilde{\chi})$ scenario, see Fig. 3. We emphasize that this indirect probe for electroweakinos via their loop contributions to the $h \rightarrow \gamma \gamma$ partial decay width is complementary to the direct searches for electroweakinos at the HL-LHC [132].

Finally, in Fig. 6 we show the HL-LHC sensitivity for the proposed new $M_{h}^{125}(\mu=-2 \mathrm{TeV})$ scenario in comparison with the $M_{h}^{125}$ scenario and the other choices of $\mu=$ $-1 \mathrm{TeV},-3 \mathrm{TeV}$, as introduced in Sect. 2. The exclusion lines and filled regions are analogous to those in Fig. 1(left), but are now determined using the HL-LHC prospective searches and measurements, instead of the current experimental results. The main qualitative features observed in Fig. 1(left) can be found here for the HL-LHC projections as well: Searches for heavy Higgs bosons in the $\tau^{+} \tau^{-}$final state cover a larger area in the $\left(M_{A}, \tan \beta\right)$ parameter plane than those in the $b \bar{b}$ final state, and the $H / A \rightarrow b \bar{b}$ search sensitivity shows a strong dependence on the size and sign of $\mu$ while there is only a moderate impact on the searches in the $\tau^{+} \tau^{-}$final state. On the other hand, Fig. 6 shows that the anticipated reach of heavy Higgs boson searches in the $b \bar{b}$ final state is competitive with the indirect reach of the anticipated Higgs-boson rate measurements. Except for $\mu=-3 \mathrm{TeV}$ the direct searches in the $b \bar{b}$ final state yield a stronger expected exclusion in the high- $M_{A}$ region than the Higgs-boson rate measurements. The flat regions towards large values of $M_{A}$ in the upper bounds on $\tan \beta$ for $\mu=-2 \mathrm{TeV}$ and $\mu=-3 \mathrm{TeV}$ are again caused by the fact that the prediction for the light Higgs-boson mass is below $122 \mathrm{GeV}$ in this region (see Fig. 1(right)), and the same applies to the lower limit in $\tan \beta$ (which is almost identical for all values of $\mu$ ). However, for $M_{A} \lesssim 2 \mathrm{TeV}$ in the scenario with $\mu=-2 \mathrm{TeV}$ and for $M_{A} \lesssim 1.5 \mathrm{TeV}$ in the scenario with $\mu=-3 \mathrm{TeV}$ the Higgs rate measurements provide sensitivity for a non-trivial upper bound on $\tan \beta$.

\section{Constraints on BSM physics from the prospective rate measurements at the HL-LHC and the ILC}

We now extend our investigations to the situation where the results from the HL-LHC are combined with prospective high-precision measurements of the Higgs signal rates at a future linear $e^{+} e^{-}$collider (for definiteness, we focus on the ILC as the currently most advanced project for which the anticipated precision levels are based on full detector simulations). We do not take into account in this context the capabilities of an $e^{+} e^{-}$linear collider for detecting new light states, like the light electroweakinos occurring in the benchmark scenarios discussed above and the possible production of additional light Higgs bosons [133-137]. The latter possibility is of less relevance in the benchmark scenarios that we discuss in the present paper, but within the MSSM context can occur in scenarios with (approximate) alignment without decoupling [10]. Light additional Higgs bosons that are compatible with present experimental constraints can occur as a generic feature in extended Higgs sectors with an additional singlet, see e.g. Refs. [22-25].

In the following we first discuss the indirect constraints on $M_{A}$ that could be inferred in the absence of a deviation from the SM predictions, i.e. under the assumption that the measured rates exactly agree with the SM prediction. It should be obvious from the discussion of the HL-LHC sensitivities in the previous section, where in the considered benchmark scenarios the prospective constraints on $M_{A}$ are already quite far in the decoupling region of the MSSM, that an improved precision of the detected Higgs-boson rates will only have a moderate effect in such a scenario. This is due to the fact that the dependence of the Higgs-boson rates on $M_{A}$ is essentially flat in this region, with only very small deviations from the SM value. It should be noted that a precision measurement of the Higgs-boson rates would have a much higher 
impact for the case, for instance, where the Higgs boson at $125 \mathrm{GeV}$ would have a non-vanishing decay mode into BSM particles. $^{12}$

In a second line of analysis we investigate scenarios that would correspond to the situation where a particular MSSM parameter point was realized in nature. We show in this context how on the basis of the Higgs rate measurements alone such a scenario could be distinguished from the SM case and how well the parameters $M_{A}$ and $\tan \beta$ could be indirectly constrained.

\subsection{Impact of the rate measurements for the case where the} $\mathrm{SM}$ is realized

In Fig. 7 we present the indirect $2 \sigma$ expected exclusion regions through precision Higgs-boson rate measurements that are obtained under the assumption that the measured rates exactly agree with the SM predictions. The results are given in the $M_{h}^{125}$ scenario (upper left panel), the $M_{h}^{125}(\tilde{\chi})$ scenario (upper right panel) and the $M_{h}^{125, \mu-}$ scenario (lower panel). We show the results for the three considered future scenarios: In light blue the HL-LHC projection is displayed, corresponding to the results already shown in Figs. 2, 3 and 6. In medium blue the projection corresponding to the combination of the HL-LHC and ILC250 measurements is shown, while the dark blue shading indicates the case where in addition also ILC500 measurements are included (see Sect. 3).

In the first two scenarios, $M_{h}^{125}$ and $M_{h}^{125}(\tilde{\chi})$, the sensitivity at large $\tan \beta$ is determined by the decoupling behavior with $M_{A}$, resulting in roughly vertical exclusion lines for $\tan \beta \gtrsim 25$ (not explicitly shown in Fig. 7). In this large$\tan \beta$ region the HL-LHC will already probe masses of the heavy Higgs bosons far in the decoupling regime, $M_{A} \gtrsim 920$ and $1000 \mathrm{GeV}$ for the $M_{h}^{125}$ and $M_{h}^{125}(\tilde{\chi})$ scenario, respectively. The ILC measurements at ILC250 and ILC500 will be able to extend the HL-LHC reach in $M_{A}$ by around $+(110-$ 125) $\mathrm{GeV}$ and $+(200-235) \mathrm{GeV}$, respectively. In the $M_{h}^{125}$ scenario, due to the absence of light SUSY particles, this lower bound on $M_{A}$ roughly remains the same for lower $\tan \beta$ values. These indirect constraints on $M_{A}$ are complementary to the sensitivity of the direct searches discussed in Fig. 2, which depend on the details of the decay patterns of the heavy Higgs bosons. The indirect constraints from the rate measurements can potentially exceed the direct search sensitivity for heavy Higgs bosons in the lower-tan $\beta$ region. In the $M_{h}^{125}$ scenario this parameter space is largely covered by the indirect constraint from confronting the prediction for

\footnotetext{
12 As mentioned above, if the mass of the lightest neutralino is below $M_{h} / 2$ the possibility of invisible Higgs decays can also be searched for directly, with an anticipated $95 \%$ CL upper limit on the branching ratio of $2.6 \%(0.3 \%)$ at the HL-LHC (for the combination of HL-LHC and ILC) $[15,107]$.
}

the mass of the light Higgs boson with the measured value (see also Fig. 2), but as discussed above this kind of constraint is highly sensitive to the details of the considered benchmark scenario.

The result for the $M_{h}^{125}(\tilde{\chi})$ scenario (upper right panel of Fig. 7) shows that there is additional sensitivity in the low$\tan \beta$ region in comparison to the case of the $M_{h}^{125}$ scenario. As already noted in the discussion of Fig. 3, the presence of light charginos in the $M_{h}^{125}(\tilde{\chi})$ scenario induces a shift in the $h \rightarrow \gamma \gamma$ partial width. If the HL-LHC measurements of the $\gamma \gamma$ rate of the Higgs boson at $125 \mathrm{GeV}$ yield exactly the SM value, a lower limit of $\tan \beta \gtrsim 12$ could be obtained almost independently of $M_{A}$. Since the $h \rightarrow \gamma \gamma$ channel is not expected to be significantly improved at the ILC, the measurements at ILC250 and ILC500 would only have a minor impact on this lower bound on $\tan \beta$ in the $M_{h}^{125}(\tilde{\chi})$ scenario. For higher values of $\tan \beta$ the ILC measurements would improve the indirect sensitivity on $M_{A}$ by about $200 \mathrm{GeV}$, similarly to the case of the $M_{h}^{125}$ scenario.

In the $M_{h}^{125, \mu-}$ scenario the situation is qualitatively different than in the other two displayed benchmark scenarios, since in this case the projected exclusion becomes stronger for increasing $\tan \beta$ values. Below $\tan \beta \sim 20$ the improvement of the expected lower $M_{A}$ limit achieved by the ILC precision is similar as in the other two scenarios. For $\tan \beta \gtrsim 20$ the indirect sensitivity up to rather high values of $M_{A}$ arises from the strong enhancement of the bottom-quark Yukawa coupling, which in turn influences all Higgs decay rates as the $h \rightarrow b \bar{b}$ partial width dominates the total decay width. In this region the main impact on constraining the parameter space at the HL-LHC arises from the $h \rightarrow \gamma \gamma$ rate measurements, since the enhancement of the bottom-quark Yukawa coupling and the corresponding increase in the total width diminishes the branching ratio into $\gamma \gamma$. The ILC measurements would add complementary information from the other branching ratios and from the production cross sections of the Higgs signal at $125 \mathrm{GeV}$.

It should be noted that the displayed results in the scenarios with negative $\mu$, see Figs. 6 and 7, can also be interpreted in the following way: the Higgs-rate measurements in the scenarios with negative $\mu$, as a consequence of their dependence on potentially large $\Delta_{b}$ corrections, provide sensitivity to set an upper bound on $|\mu|$ (assuming that $\mu<0$ holds) depending on $M_{A}$ and $\tan \beta$. As an example, measuring SM Higgs rates at the HL-LHC would exclude the point $M_{A}=1300 \mathrm{GeV}$ and $\tan \beta=20$ for $\mu=-3 \mathrm{TeV}$ but not for $\mu=-2 \mathrm{TeV}$. While this sensitivity is restricted to a certain range of $M_{A}$ (see the discussion of Fig. 6) and relies on the assumption $\mu<0$, it could nevertheless be of interest in the context of future direct searches for supersymmetric particles. We come back to the discussion of the influence of the bottom-quark Yukawa coupling enhancement on the 

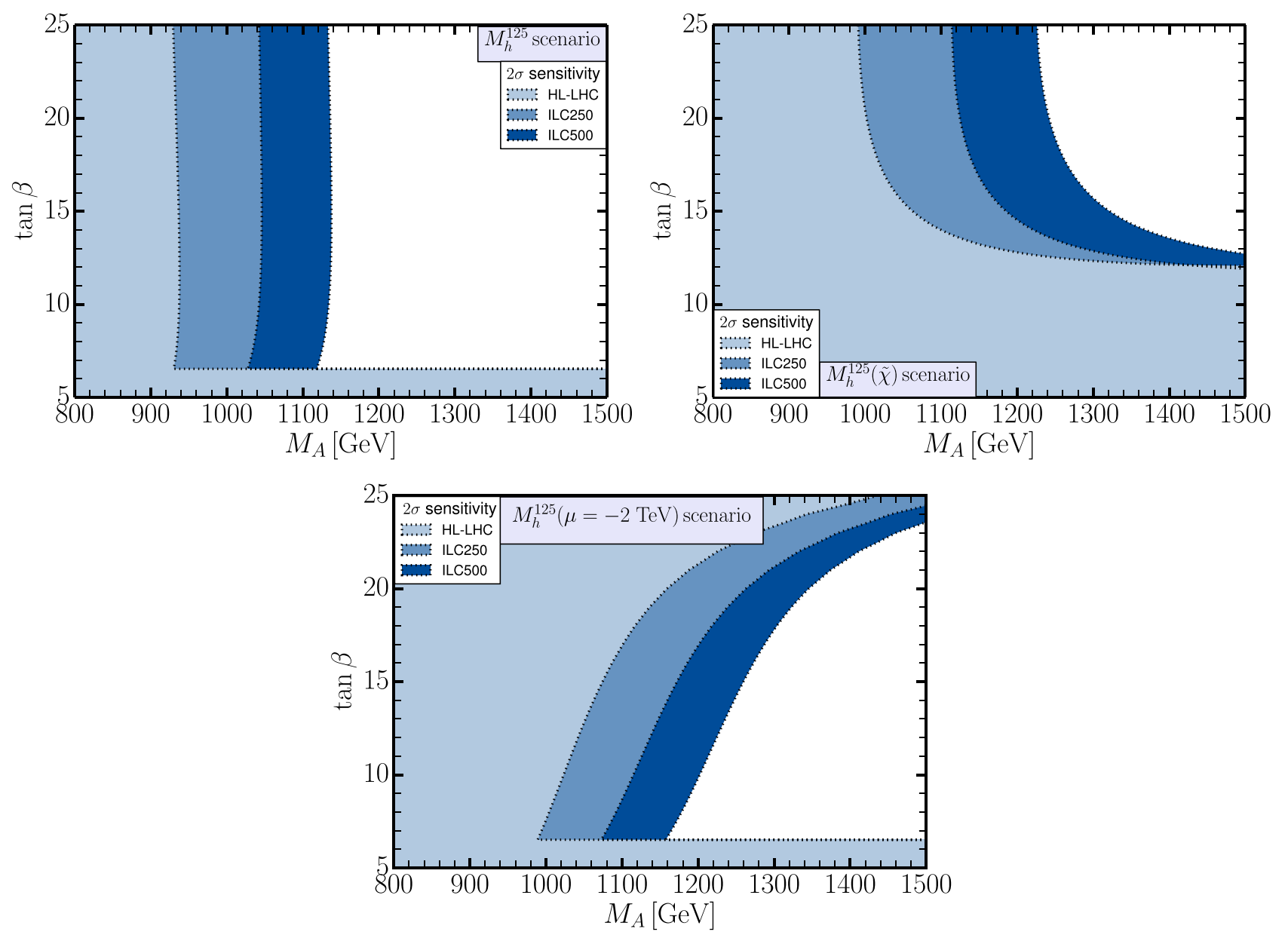

Fig. 7 Prospective $2 \sigma$ indirect exclusion region from Higgs rate measurements at the HL-LHC and the ILC, assuming agreement with the SM predictions, in the $M_{h}^{125}$ scenario (upper left panel), the $M_{h}^{125}(\tilde{\chi})$ scenario (upper right panel), and the $M_{h}^{125, \mu-}$ scenario (bottom panel)

Higgs rates in the next subsection, however at a lower value of $\tan \beta$, where the effect is not as pronounced, see Fig. 10 below.

As stressed above, the analysis within specific benchmark scenarios, where besides $M_{A}$ and $\tan \beta$ all other SUSY parameters are fixed to specific values by definition, and the assumption used in this section that the detected rates exactly agree with the SM predictions, cannot demonstrate the full potential of the precision measurements of the Higgs boson rates at the HL-LHC and the ILC. In particular, assuming that the underlying nature of the probed physics scenario is the MSSM implies very important correlations between the couplings, the production and decay rates of the Higgs boson at $125 \mathrm{GeV}$. Thus, a precise measurement of only few observables is already sufficient to determine the decoupling behavior if the underlying structure of the physics scenario is assumed to be known. Instead, if no such assumption is made the full breadth of precision measurements at the HLLHC and the ILC will be crucial in order to either determine the nature of observed patterns of deviations from the SM or to set constraints on wide classes of possible extensions or alternatives to the SM. Specifically, the model-independent measurement of the total Higgs production cross section of $e^{+} e^{-} \rightarrow Z h$ at the ILC (which allows a model-independent determination of the Higgs branching ratios and provides a robust method for obtaining the total width with high precision) has no direct impact in our benchmark scenarios, while it is crucial for probing models that both allow for additional Higgs boson decay mode(s) as well as a compensating enhancement of the production rates [138].

Furthermore, the assumption that all future HL-LHC and ILC measurements of Higgs production and decay modes will yield exact agreement with the SM predictions is of course not realistic. Even in the absence of any contribution of new physics one would still expect that the measured values are scattered around the SM values according to their statistical uncertainty. In order to answer the question whether the actually observed pattern of measurements with a certain amount of data hints at a non-zero deviation from the SM or not, the full set of observables accessible at the HL-LHC and 

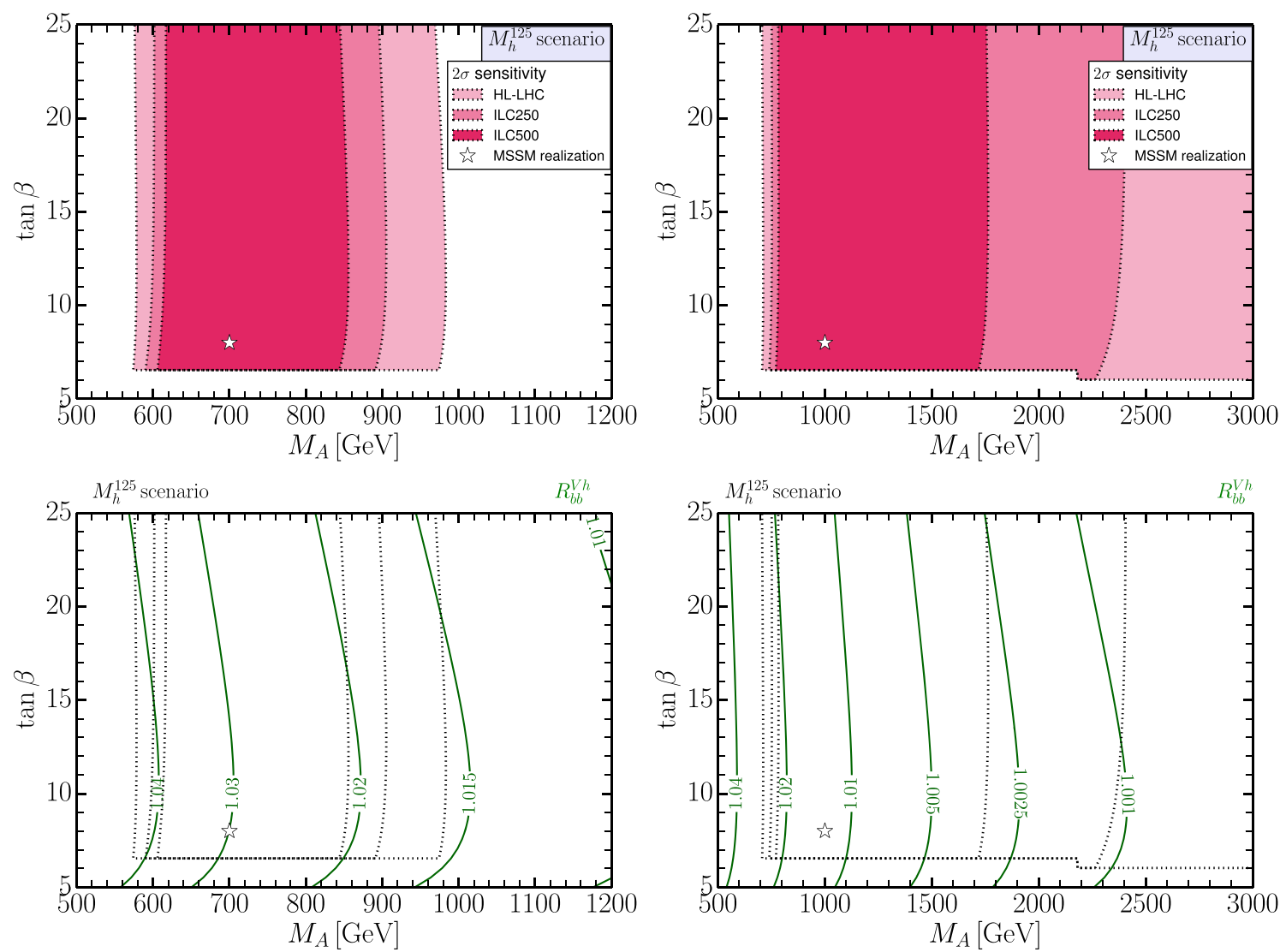

Fig. 8 Upper panels: Indirect $2 \sigma$ constraints in the $\left(M_{A}, \tan \beta\right)$ parameter plane of the $M_{h}^{125}$ scenario from prospective Higgs-boson signalrate measurements at the HL-LHC (faint red) and in combination with ILC250 (medium red) and ILC500 (bright red) measurements, assuming that the point, indicated by a star, $\left(M_{A}, \tan \beta\right)=(700 \mathrm{GeV}, 8)$ (left

panel) or $\left(M_{A}, \tan \beta\right)=(1 \mathrm{TeV}, 8)$ (right panel) is realized in nature. Lower panels: SM-normalized Higgs rate in the $p p \rightarrow V h, h \rightarrow b \bar{b}$ channel, $R_{b b}^{V h}$ (green contours), with the $2 \sigma$ parameter ranges from the upper panels superimposed

the ILC measured with the highest possible accuracy will be instrumental.

\subsection{Impact of the rate measurements for the case where a particular MSSM scenario is realized}

We now take a different perspective and assume that future precision Higgs-boson rate measurements reveal deviations from the SM prediction, which are compatible with MSSM predictions for the Higgs-boson rates. Specifically, we assume that a certain parameter point within the considered MSSM benchmark scenario is realized and set the central values of all projected HL-LHC and ILC measurements to the predicted rates at this parameter point (while keeping the same relative uncertainty as for the SM case). We then again analyze how well the MSSM parameter space can be indirectly constrained from precision measurements of the rates of the Higgs boson at $125 \mathrm{GeV} .{ }^{13}$ We first assume the

\footnotetext{
13 An earlier analysis of this type can be found in Ref. [139], see also Ref. [140].
}

realization of MSSM parameter points at a moderate value of $\tan \beta$ that are expected to elude a direct $5 \sigma$ discovery in heavy Higgs boson searches. The chosen points lie close to the border of the $2 \sigma$ sensitivity of HL-LHC direct searches in the $\tau^{+} \tau^{-}$channel. Furthermore we briefly discuss the case that a relatively large value of $\tan \beta$ could be realized in nature. In light of the existing search limits this would mean that the associated value of $M_{A}$ has to be significantly higher, and correspondingly the impact of such a scenario on the rates of the Higgs boson at $125 \mathrm{GeV}$ is expected to be rather small.

We start with the $M_{h}^{125}$ scenario in Fig. 8, where we assume in the left panels $\left(M_{A}, \tan \beta\right)=(700 \mathrm{GeV}, 8)$ and in the right panels $\left(M_{A}, \tan \beta\right)=(1000 \mathrm{GeV}, 8)$ being realized in nature. In fact, both points will be probed at the $\gtrsim 2 \sigma$ level by heavy Higgs searches in the $\tau^{+} \tau^{-}$final state at the HL-LHC, see Fig. 2, which would thus give complementary information. Here we want to focus on the indirect constraints on the parameters that can be obtained from the precision rate measurements of the Higgs boson at $125 \mathrm{GeV}$. In the upper panels of Fig. 8 we show the parameter regions which are 
preferred at the $2 \sigma$ level by the HL-LHC alone (faint red), and in combination with the ILC250 measurements (medium red) and the ILC500 measurements (dark red). The lower panels of Fig. 8 show contours for $R_{b b}^{V h}$, i.e. the Higgs boson signal rate for $p p \rightarrow V h\left(V=W^{ \pm}, Z\right)$ production, followed by the decay $h \rightarrow b \bar{b}$, and normalized to its SM prediction (at the same Higgs boson mass). In the considered parameter space $R_{b b}^{V h}$ is very similar to the SM-normalized rates of the ILC processes $e^{+} e^{-} \rightarrow Z h, h \rightarrow b \bar{b}$ and $e^{+} e^{-} \rightarrow \nu \bar{v} h, h \rightarrow$ $b \bar{b}$, as the $g g \rightarrow Z h$ contribution to $R_{b b}^{V h}$ is subdominant, and the rates of the other production processes contributing to $R_{b b}^{V h}$ scale uniformly with the squared $h V V$ coupling. This signal process is the most sensitive search channel for the $b \bar{b}$ final state, with an anticipated HL-LHC (ATLAS and CMS combined) precision for the signal strength of around $4.6 \%$ in the $p p \rightarrow Z h$ production mode [15].

As already discussed above, the Higgs rate measurements have almost no sensitivity on $\tan \beta$ in the $M_{h}^{125}$ scenario (in the range $\tan \beta \gtrsim 6$ where in this benchmark scenario the predicted value of the mass of the light Higgs boson is compatible with the experimental value of the detected Higgs signal). For the assumed point $\left(M_{A}, \tan \beta\right)=(700 \mathrm{GeV}, 8)$ the heavy Higgs mass scale, $M_{A}$, could be constrained by prospective Higgs rate measurements to

$$
\begin{aligned}
575 \mathrm{GeV} & \lesssim M_{A} \lesssim 980 \mathrm{GeV} & & (\text { HL-LHC }) \\
600 \mathrm{GeV} & \lesssim M_{A} \lesssim 900 \mathrm{GeV} & & (+ \text { ILC250) } \\
615 \mathrm{GeV} & \lesssim M_{A} \lesssim 850 \mathrm{GeV} & & (+ \text { ILC500) }
\end{aligned}
$$

at the $2 \sigma$ level. In contrast, assuming the point closer to the decoupling limit, $\left(M_{A}, \tan \beta\right)=(1000 \mathrm{GeV}, 8)$, the expected $2 \sigma$ ranges are

$$
\begin{aligned}
700 \mathrm{GeV} & \lesssim M_{A} \quad(\mathrm{HL}-\mathrm{LHC}) \\
750 \mathrm{GeV} & \lesssim M_{A} \lesssim 2400 \mathrm{GeV} \quad(+\mathrm{ILC} 250), \\
780 \mathrm{GeV} & \lesssim M_{A} \lesssim 1750 \mathrm{GeV} \quad(+ \text { ILC500) }
\end{aligned}
$$

In this case the prospective accuracy of the signal strength measurements at the HL-LHC is not sufficient to place an indirect upper bound on $M_{A}$ at the $2 \sigma$ level. The incorporation of the precision measurements at the ILC would significantly improve the sensitivity of the Higgs rate measurements for a distinction between the SM and the MSSM. The possibility to establish an upper bound on $M_{A}$ via the rate measurements of the Higgs state at $125 \mathrm{GeV}$ at the HL-LHC and the ILC would provide a clear target for the direct searches for additional Higgs bosons.

The bottom panels in Fig. 8 show that in the $2 \sigma$-allowed region, the deviations of $R_{b b}^{V h}$ are $\lesssim 2 \%$ for the case where $M_{A}=1000 \mathrm{GeV}$ has been assumed and $\lesssim 4 \%$ for the case with $M_{A}=700 \mathrm{GeV}$. Accordingly, the future HL-LHC measurements of the $p p \rightarrow Z h, h \rightarrow b \bar{b}$ rate have only a limited sensitivity for constraining the parameter space in the considered scenarios. On the other hand, the inclusive rate measurements in the $h \rightarrow \gamma \gamma, h \rightarrow V V\left(V=W^{ \pm}, Z\right)$ and $h \rightarrow \tau^{+} \tau^{-}$channels at the HL-LHC have sensitivity to modifications of the $h b \bar{b}$ coupling through the dependence of the involved branching ratio on the total width of the Higgs boson at $125 \mathrm{GeV}$. For instance, near the assumed points $\left(M_{A}, \tan \beta\right)=(700 \mathrm{GeV}, 8)$ and $(1000 \mathrm{GeV}, 8)$, the Higgsto-diphoton rate is suppressed by $7 \%$ and $3 \%$ with respect to the SM prediction, respectively, as a result of a slightly enhanced bottom-quark Yukawa coupling and its impact on the total Higgs width. The combination of the measurements of the Higgs signal rates at the HL-LHC in various channels involving the product of the production cross sections and decay branching ratios will therefore provide sensitive information on possible deviations from the SM, while it will be non-trivial to disentangle the source of the deviations. Concerning the prospective rate measurements at the ILC, the most precise Higgs rate measurements will be performed in the $e^{+} e^{-} \rightarrow Z h, h \rightarrow b \bar{b}$ channel during the run at $250 \mathrm{GeV}$ and in the $e^{+} e^{-} \rightarrow \nu \bar{\nu} h, h \rightarrow b \bar{b}$ channel in the $500 \mathrm{GeV}$ run [107], each with a precision at the sub-percent level. The ILC measurements will therefore complement the information obtainable at the HL-LHC with high-precision input on observables that cannot be well exploited at the LHC. The ILC will furthermore provide model-independent measurements of individual branching ratios. This kind of information will be crucial in order to determine the source of possible deviations without invoking model assumptions. In order to investigate the underlying nature of detected deviations from the SM, the indirect constraints that we have discussed here should of course be applied in the context of the information that is available from the direct searches for additional Higgs bosons (see in particular Fig. 2) and other states of new physics. The limits from these searches may in fact exclude large regions of the parameter space that is favored by the indirect constraints. Naturally, in case of a significant excess (or more than one) in the direct searches the prospects for narrowing down the possible nature of new physics with the combination of direct and indirect information would of course much improved.

The pattern of the deviations from the SM predictions corresponding to the situation where the parameter point $\left(M_{A}, \tan \beta\right)=(700 \mathrm{GeV}, 8)$ or the point $\left(M_{A}, \tan \beta\right)=$ $(1000 \mathrm{GeV}, 8)$ of the $M_{h}^{125}$ scenario is realized in nature is shown in Fig. 9. The displayed plots, which we denote as "Wäscheleinen-plots" (washing line plots) in the following, show the predicted light Higgs couplings (normalized to the SM prediction) at the assumed MSSM points in the $\kappa$ framework [63], along with the anticipated $1 \sigma$ precision of a rather general $\kappa$ determination [107] from prospective Higgs rate measurements at the HL-LHC and the ILC. ${ }^{14}$ It should

\footnotetext{
${ }^{14}$ More specifically, we show the expected precision of the $\kappa$ parameters in the kappa-3 scenario [107].
} 


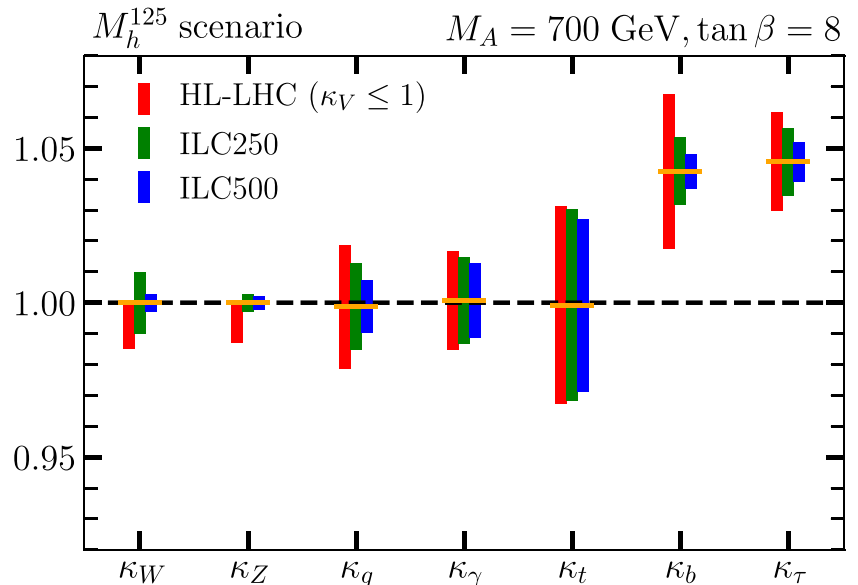

Fig. 9 Wäscheleinen-plots for the two assumed MSSM parameter points $\left(M_{A}, \tan \beta\right)=(700 \mathrm{GeV}, 8)$ (left) and $\left(M_{A}, \tan \beta\right)=$ $(1 \mathrm{TeV}, 8)$ (right) in the $M_{h}^{125}$ scenario: Predicted Higgs couplings in the $\kappa$ framework (orange horizontal bars) along with the anticipated

be noted that the coupling determination at the HL-LHC is based on the theoretical assumption $\kappa_{V} \leq 1$, while no such assumption is needed for the coupling determinations at the ILC. The plots in Fig. 9 show that for the assumed parameter points sizable deviations from the SM only occur for the bottom-quark and tau-lepton Yukawa couplings, represented by $\kappa_{b}$ and $\kappa_{\tau}$, respectively. At the HL-LHC the precision of the $\kappa_{b}$ and $\kappa_{\tau}$ determination is at the 2 and $1.5 \%$ level, respectively. One can see that in particular for the assumed point $\left(M_{A}, \tan \beta\right)=(1000 \mathrm{GeV}, 8)$ the ILC accuracy will be crucial in order to experimentally establish a significant deviation of the rates of the Higgs boson at $125 \mathrm{GeV}$ from the SM predictions. Moreover, the ILC measurements will provide a crucial consistency test of the correlations between couplings, for instance, between $\kappa_{b}$ and $\kappa_{\tau}$, and will therefore help to further discriminate between different BSM interpretations.

The prospects for the indirect constraints from the Higgs rate measurements at the HL-LHC and ILC for the assumed parameter points $\left(M_{A}, \tan \beta\right)=(700 \mathrm{GeV}, 8)$ and $\left(M_{A}\right.$, $\tan \beta)=(1000 \mathrm{GeV}, 8)$ in the $M_{h}^{125, \mu-}$ scenario are shown in the upper panels of Fig. 10, while the lower panels show the corresponding predictions for the (SM-normalized) Higgs rate for the $p p \rightarrow V h, h \rightarrow b \bar{b}$ channel, $R_{b b}^{V h}$. The main effect of changing from $\mu=+1 \mathrm{TeV}$ (the value chosen in the previously discussed $M_{h}^{125}$ scenario) to $\mu=-2 \mathrm{TeV}$ is the non-trivial $\tan \beta$-dependence of the preferred parameter region. In the case of the first assumed point $\left(M_{A}, \tan \beta\right)=$ $(700 \mathrm{GeV}, 8)$ (left panels) the Higgs rate measurements at the HL-LHC (and including the measurements at ILC250 and ILC500) yield an upper limit of $\tan \beta \lesssim 27(\tan \beta \lesssim$ $26,24.5$, respectively). These upper limits become significantly stronger at smaller $M_{A}$ values. On the other hand,

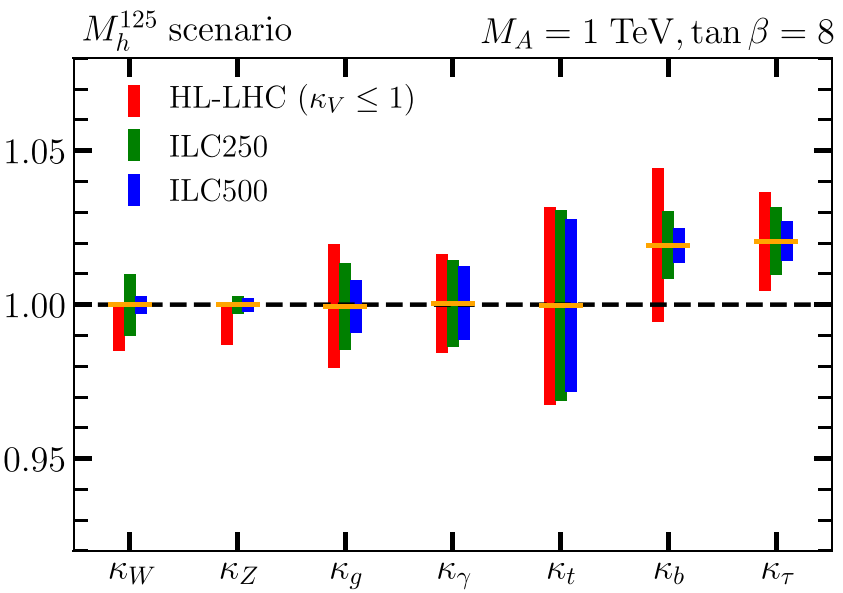

$1 \sigma$ precision from a global fit [107] to Higgs rate measurements at the HL-LHC, where the theoretical assumption $\kappa_{V} \leq 1$ is employed, and including prospective measurements at ILC250 and ILC500 (but without imposing an assumption on $\kappa_{V}$ )

upper bounds on $M_{A}$ from the Higgs rate measurements can be inferred at the largest values of $\tan \beta$, namely $M_{A} \lesssim$ $1420 \mathrm{GeV}$ at the HL-LHC, $M_{A} \lesssim 1100 \mathrm{GeV}$ including measurements at ILC250, and $M_{A} \lesssim 980 \mathrm{GeV}$ including measurements at ILC500. For the plots showing the indirect constraints for the case of the other assumed parameter point $\left(M_{A}, \tan \beta\right)=(1000 \mathrm{GeV}, 8)$ (right panels), there is a cutoff visible for $M_{A} \gtrsim 1400 \mathrm{GeV}$ that restricts the displayed values of $\tan \beta$ to $\tan \beta \lesssim 27$ (this feature is not visible in the left panels due to the smaller displayed range of $M_{A}$ ). This cutoff is not a consequence of the rate measurements but arises in this benchmark scenario because of the incompatibility of the light Higgs mass with the observed value, as discussed above (see the right panel of Fig. 1). The impact of the Higgs rate measurements for constraining $\tan \beta$ can be seen in this case for $M_{A} \lesssim 1400 \mathrm{GeV}$. For the assumed parameter point $\left(M_{A}, \tan \beta\right)=(1000 \mathrm{GeV}, 8)$ an indirect upper limit on $M_{A}$ from the Higgs rate measurements can only be obtained using the ILC measurements. Here the measurements from later ILC stages (ILC500) will sharpen the upper limits on $M_{A}$ obtained from using the measurements at the initial ILC run (ILC250) by around $400 \mathrm{GeV}$, independently of the $\tan \beta$ value. For both assumed parameter points the incorporation of the Higgs rate measurements at the ILC leads to a large reduction of the allowed parameter space in the $\left(M_{A}, \tan \beta\right)$ plane.

The pattern of the deviations in $R_{b b}^{V h}$ in the preferred regions of the $\left(M_{A}, \tan \beta\right)$ plane (lower panels of Fig. 10) is similar to the case of Fig. 8. Thus, also in this case the incorporation of the ILC measurements would lead to a much larger set of observables showing sizable deviations from the SM. This kind of information will be crucial to determine the underlying nature of the detected deviations. As 

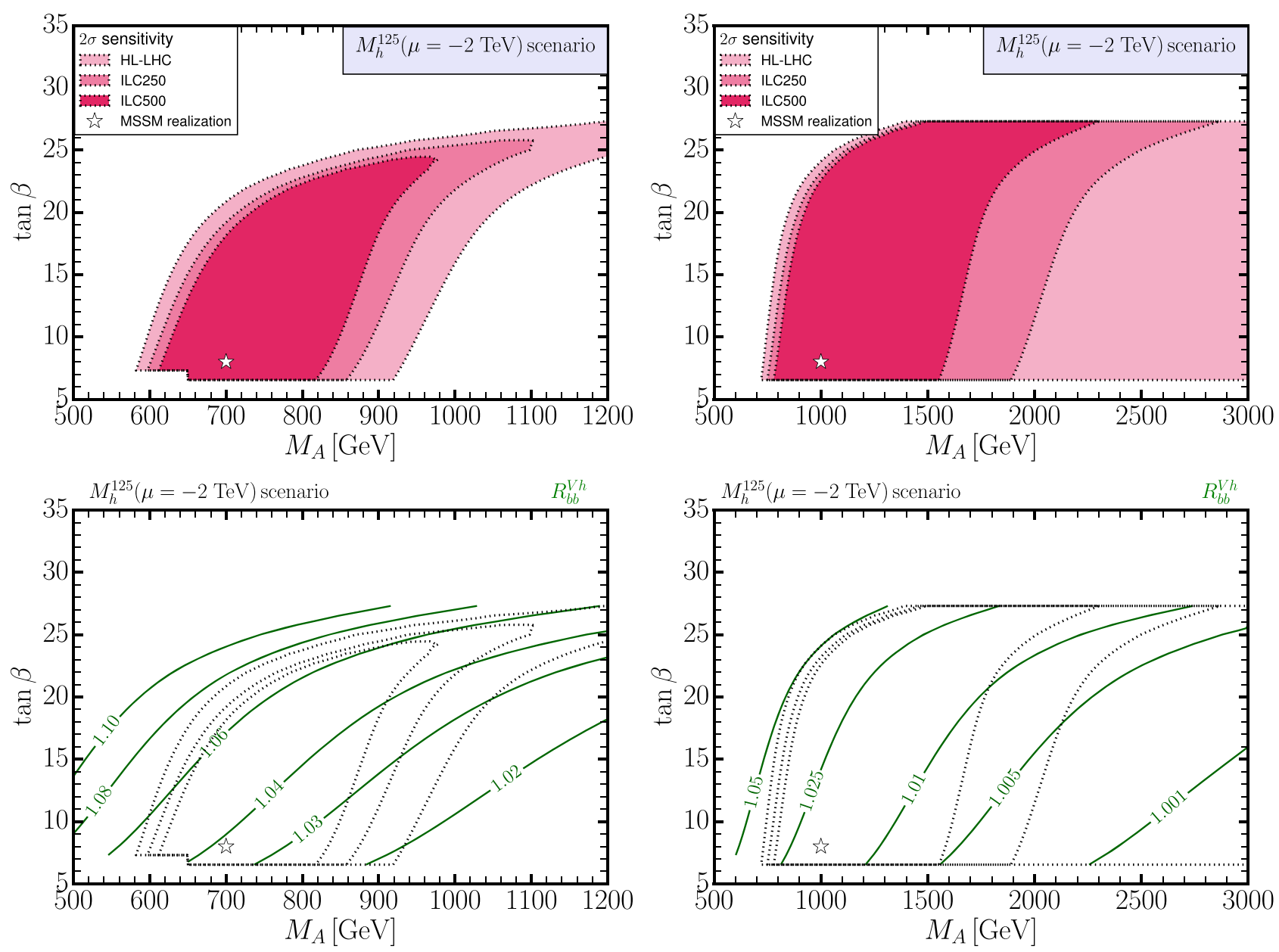

Fig. 10 Indirect $2 \sigma$ constraints in the $\left(M_{A}, \tan \beta\right)$ parameter plane from prospective Higgs-boson signal-rate measurements at the HLLHC and the ILC (upper row) and $R_{b b}^{V h}$ contours (lower row) in the $M_{h}^{125, \mu-}$ scenario,assuming that the point, indicated by a star,

$\left(M_{A}, \tan \beta\right)=(700 \mathrm{GeV}, 8)$ (left panels) or $\left(M_{A}, \tan \beta\right)=(1 \mathrm{TeV}, 8)$ (right panels) is realized in nature. The same color coding as in Fig. 8 is used

discussed above, those investigations should of course be based on both the direct information from searches and the indirect constraints. For the $M_{h}^{125, \mu-}$ scenario large parts of the parameter region that would be preferred by the prospective Higgs rate measurements are within the $2 \sigma$ reach of heavy Higgs searches in the $\tau^{+} \tau^{-}$and possibly even $b \bar{b}$ final states at the HL-LHC, see Fig. 6. A robust excess in these searches would provide clues for the mass scale of the heavy Higgs bosons, $M_{A}$. The $125 \mathrm{GeV}$ Higgs rate measurements could then, together with first potential measurements of the strength of such a heavy Higgs boson signal, allow one to put new physics interpretations under scrutiny and, within the considered scenario, lead to strongly improved constraints on the model parameters.

In Fig. 11 we show Wäscheleinen-plots for the parameter points $\left(M_{A}, \tan \beta\right)=(700 \mathrm{GeV}, 8)$ (upper left panel) and $\left(M_{A}, \tan \beta\right)=(1000 \mathrm{GeV}, 8)$ (upper right panel) in the

$M_{h}^{125, \mu-}$ scenario, i.e. we show the predicted Higgs couplings represented by $\kappa$ scale factors in the displayed scenarios along with the prospective $1 \sigma$ precision levels of their determination from a global fit [107] to Higgs rate measurements. For the precisions from HL-LHC the theoretical assumption $\kappa_{V} \leq 1$ is employed, while including prospective measurements at ILC250 and ILC500 the assumption on $\kappa_{V}$ is dropped. The lower panel shows the comparison between the predicted Higgs couplings $\kappa_{b}$ for the assumed point $\left(M_{A}, \tan \beta\right)=(1 \mathrm{TeV}, 8)$ and the anticipated experimental precisions for different values of $\mu$. Besides the predictions for different MSSM parameters also the prediction in the THDM-II for $\left(M_{A}, \tan \beta\right)=(1 \mathrm{TeV}, 8)$ is indicated (obtained approximately from the MSSM predictions as described below). The results for the $M_{h}^{125, \mu-}$ scenario displayed in the upper panels of Fig. 11 are both qualitatively and quantitatively similar to the $M_{h}^{125}$ scenario as shown in 

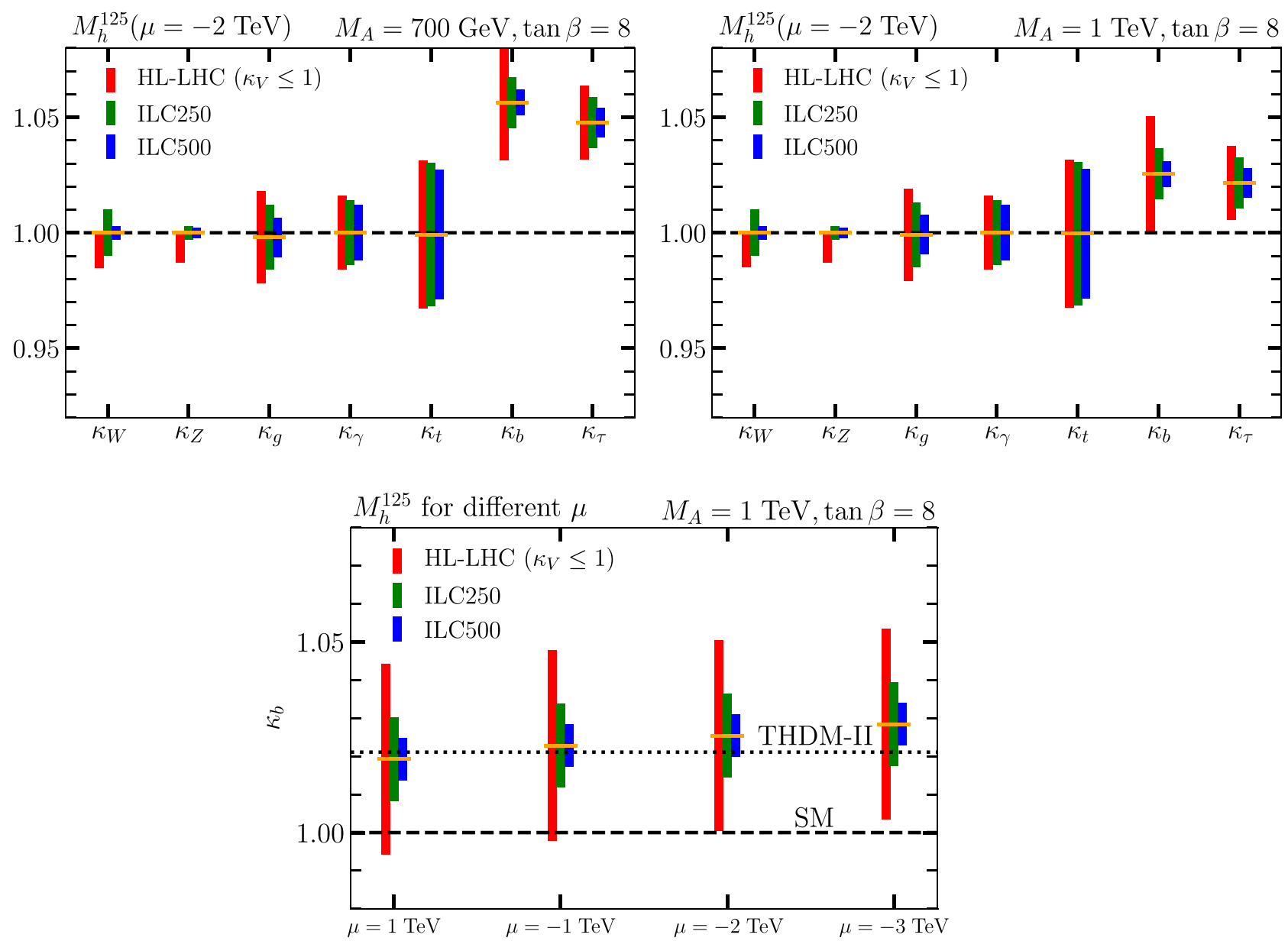

Fig. 11 Wäscheleinen-plots, using the same color coding as in Fig. 9, for the two assumed MSSM parameter points $\left(M_{A}, \tan \beta\right)=$ (700 GeV, 8) (upper left panel) and $\left(M_{A}, \tan \beta\right)=(1 \mathrm{TeV}, 8)$ (upper right panel) in the $M_{h}^{125, \mu-}$ scenario. The lower panel shows for the assumed point $\left(M_{A}, \tan \beta\right)=(1 \mathrm{TeV}, 8)$ and different values of $\mu$ the prospects for $\kappa_{b}$, where for comparison also the corresponding predic- tion in the THDM-II (see text) is indicated (dotted line), see text for details. The Higgs couplings in the $\kappa$ framework predicted in the displayed scenarios are compared with the anticipated $1 \sigma$ precision from Higgs rate measurements, where at the HL-LHC the theoretical assumption $\kappa_{V} \leq 1$ is employed, while for the results including prospective measurements at ILC250 and ILC500 no assumption on $\kappa_{V}$ is employed
Fig. 9. As expected, the value of $\mu=-2 \mathrm{TeV}$ in the $M_{h}^{125, \mu-}$ scenario leads to larger deviations in $\kappa_{b}$ and $\kappa_{\tau}$ from the SM value in comparison to the case of $\mu=+1 \mathrm{TeV}$ adopted in the $M_{h}^{125}$ scenario. This is most visible in the shift of $\kappa_{b}$ for the assumed point $\left(M_{A}, \tan \beta\right)=(700 \mathrm{GeV}, 8)$ (upper left panel) in comparison to the left plot of Fig. 9. The deviations from the SM predictions are smaller for the assumed point $\left(M_{A}, \tan \beta\right)=(1000 \mathrm{GeV}, 8)$, and also the impact of different choices for $\mu$ is smaller in this case. This is illustrated for $\kappa_{b}$ in the lower panel of Fig. 11 for $\left(M_{A}, \tan \beta\right)=$ $(1000 \mathrm{GeV}, 8)$ and $\mu=+1,-1,-2,-3 \mathrm{TeV}$. The variation of the $\mu$ values via the $\Delta_{b}$ corrections yields upward (downward) shifts of $\kappa_{b}$ for negative (positive) values of $\mu$ which in total amount to modifications of up to $1 \%$. Accordingly, while the induced variation of the genuine SUSY loop contribution $\Delta_{b}$ (see Eq. 1) can be relatively large, for $M_{A}=1 \mathrm{TeV}$ these effects are already heavily suppressed as a consequence of the decoupling properties of the MSSM. The behavior of $\kappa_{b}$ for $M_{A}=1 \mathrm{TeV}$ is therefore mainly governed by the lowest-order mixing of the two Higgs doublets. Indeed, the observed deviation from the SM value in $\kappa_{b}$ of about $2 \%$ for $M_{A}=1 \mathrm{TeV}$ is in agreement with the expected behavior arising from the tree-level prediction, $\kappa_{b} \approx 1+2 \frac{M_{Z}^{2}}{M_{A}^{2}}$. The dotted horizontal line in the lower panel of Fig. 11 indicates the prediction for the THDM type II, disregarding any additional SUSY effect beyond the mixing of the $\mathcal{C P}$-neutral Higgs bosons (obtained approximatively by averaging between the MSSM prediction for $\mu=-1 \mathrm{TeV}$ ( $\Delta_{b}$-enhanced) and the MSSM prediction for $\mu=+1 \mathrm{TeV}$ ( $\Delta_{b}$-suppressed) in the $M_{h}^{125}$ scenario). The comparison of the $\kappa_{b}$ value for the THDM-II with the variation of $\kappa_{b}$ and $\kappa_{\tau}$ in the MSSM shows that for $M_{A} \gtrsim 1 \mathrm{TeV}$ the prospects for distinguish- 

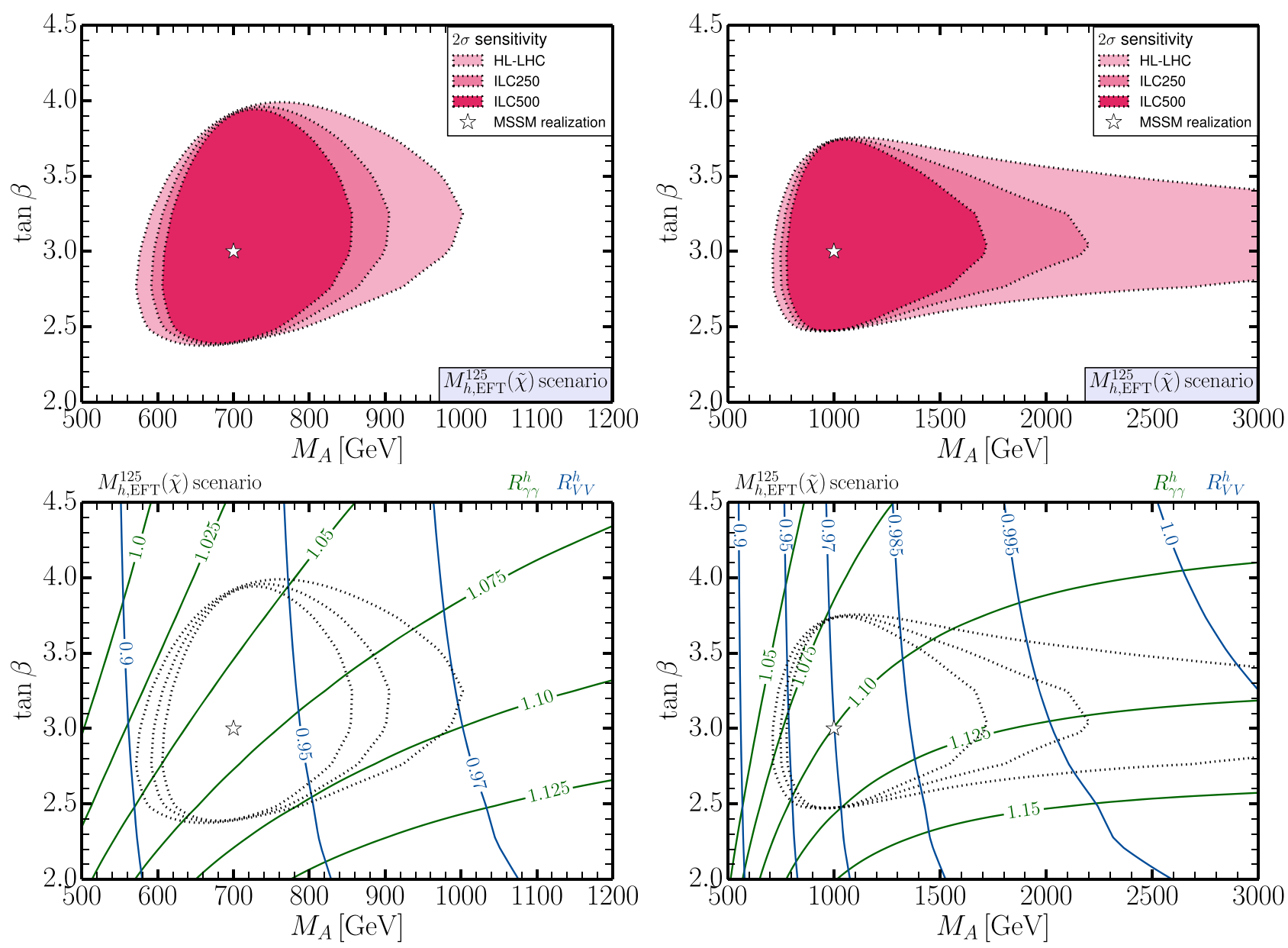

Fig. 12 Indirect $2 \sigma$ constraints in the $\left(M_{A}, \tan \beta\right)$ parameter plane of the $M_{h \text { EFT }}^{125}(\tilde{\chi})$ scenario from prospective Higgs-boson signal-rate measurements at the HL-LHC and the ILC (upper row) and contours for SM-normalized Higgs rates (lower row) with the same color coding as in Fig. 8, assuming that the point, indicated by a star, $\left(M_{A}, \tan \beta\right)=$

ing between the MSSM and a THDM-II via the differences in the higher-order contributions to $\kappa_{b}$ and $\kappa_{\tau}$ do not appear to be promising. We note this point since parameter points that have been used elsewhere in the literature seem to indicate that such a distinction could be possible. ${ }^{15}$

We now turn to the $M_{h \text { EFT }}^{125}(\tilde{\chi})$ scenario where we consider as possible realizations the points $\left(M_{A}, \tan \beta\right)=$ $(700 \mathrm{GeV}, 3)$ and $\left(M_{A}, \tan \beta\right)=(1000 \mathrm{GeV}, 3)$. In comparison to the previously discussed points in the $M_{h}^{125}$ and $M_{h}^{125, \mu-}$ scenarios (as well as its variations with different values of $\mu$ ), these parameter points are more difficult to probe directly by experimental searches for heavy Higgs bosons, since at such low values of $\tan \beta$ the $p p \rightarrow H / A \rightarrow$ $\tau^{+} \tau^{-}$rate does not contain a large enhancement factor.

\footnotetext{
$\overline{15}$ The pMSSM example point "(1)" that was used in Ref. [141] has meanwhile been found to be invalid due to corrected theory calculations.
}

(700 GeV, 3) (left panel) or $\left(M_{A}, \tan \beta\right)=(1 \mathrm{TeV}, 3)$ (right panel) is realized in nature. The blue and green contours in the lower panels show the inclusive rate for $p p \rightarrow h \rightarrow V V\left(V=W^{ \pm}, Z\right), R_{V V}^{h}$, and the inclusive rate for $p p \rightarrow h \rightarrow \gamma \gamma, R_{\gamma \gamma}^{h}$, respectively, with the $2 \sigma$ parameter ranges from the upper panels superimposed

Other direct heavy Higgs searches, in particular in the ditop final state and in electroweakino final states as suggested in Refs. $[10,11,123]$ could improve the sensitivity. Moreover, as discussed above, LHC searches for direct production of mass-compressed electroweakinos have the potential to probe a scenario with such low values of $\tan \beta$ in the electroweakino sector and can thus provide complementary information [132].

In Fig. 12 we show the results in the $M_{h, \mathrm{EFT}}^{125}(\tilde{\chi})$ scenario, with the assumed parameter point $\left(M_{A}, \tan \beta\right)=$ $(700 \mathrm{GeV}, 3)$ in the left panels, and $\left(M_{A}, \tan \beta\right)=(1000$ $\mathrm{GeV}, 3)$ in the right panels. The expected $2 \sigma$ allowed parameter ranges obtained by Higgs-boson signal-rate measurements are shown in the upper panel of Fig. 12 with the same color coding as before in Figs. 8 and 10. The indirect bounds from the Higgs rate measurements on $M_{A}$ for the first 


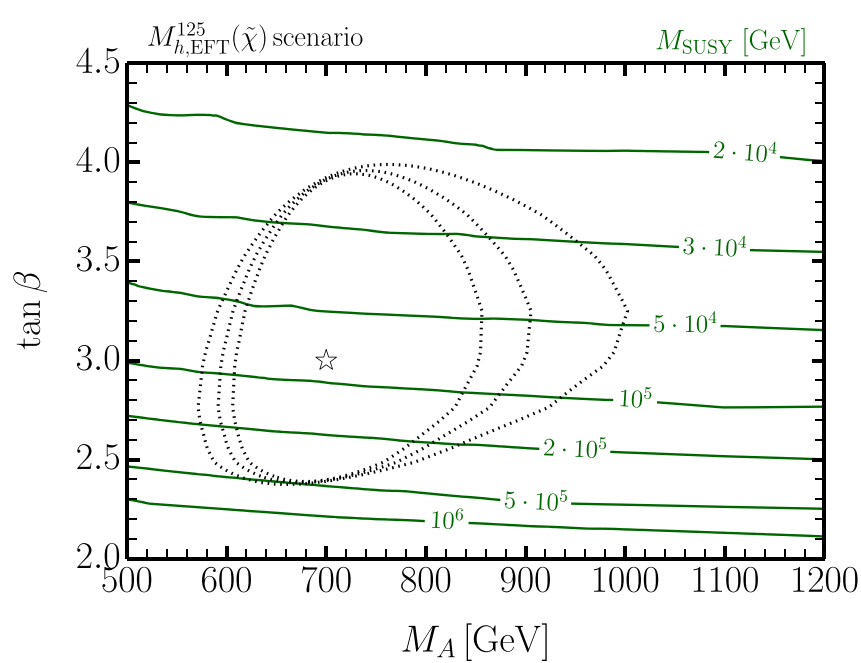

Fig. 13 Contours of the scalar fermion soft-SUSY breaking mass $M_{\mathrm{SUSY}}$ in the $\left(M_{A}, \tan \beta\right)$ parameter plane of the $M_{h \mathrm{EFT}}^{125}(\tilde{\chi})$ scenario discussed in Fig. 12, assuming that the point, indicated by a star, $\left(M_{A}, \tan \beta\right)=(700 \mathrm{GeV}, 3)$ (left panel) or $\left(M_{A}, \tan \beta\right)=(1 \mathrm{TeV}, 3)$

assumed point at $\left(M_{A}, \tan \beta\right)=(700 \mathrm{GeV}, 3)$ amount to

$$
\begin{aligned}
& 575 \mathrm{GeV} \lesssim M_{A} \lesssim 1000 \mathrm{GeV} \quad \text { (HL-LHC) } \\
& 590 \mathrm{GeV} \lesssim M_{A} \lesssim 900 \mathrm{GeV} \quad(+ \text { ILC250) }, \\
& 610 \mathrm{GeV} \lesssim M_{A} \lesssim 850 \mathrm{GeV} \quad \text { (+ILC500), }
\end{aligned}
$$

whereas for the second assumed point, $\left(M_{A}, \tan \beta\right)=$ $(1000 \mathrm{GeV}, 3)$, we find

$$
\begin{aligned}
700 \mathrm{GeV} & \lesssim M_{A} & & (\text { HL-LHC) } \\
750 \mathrm{GeV} & \lesssim M_{A} \lesssim 2200 \mathrm{GeV} & & (+\mathrm{ILC} 250), \\
780 \mathrm{GeV} & \lesssim M_{A} \lesssim 1710 \mathrm{GeV} & & (+\mathrm{ILC} 500)
\end{aligned}
$$

These $M_{A}$ ranges are very similar to those found in the $M_{h}^{125}$ scenario for the two assumed points at the same $M_{A}$ values. In particular, for the assumed point with $M_{A}=1 \mathrm{TeV}$ the prospective accuracy of the signal strength measurements at the HL-LHC is not sufficient to place an indirect upper bound on $M_{A}$ at the $2 \sigma$ level. As in the previous scenarios, the ILC measurements would be essential in this case to obtain an upper bound on $M_{A}$. In contrast to the scenarios discussed above, however, for the assumed value of $\tan \beta=3$ in this scenario with light charginos the Higgs rate measurements would not only provide a high sensitivity for a distinction from the SM but would also allow one to constrain $\tan \beta$ to a narrow range

$$
\begin{aligned}
& 2.5 \leq \tan \beta \leq 4.0 \text { for }\left(M_{A}, \tan \beta\right)=(700 \mathrm{GeV}, 3), \\
& 2.5 \leq \tan \beta \leq 3.75 \text { for }\left(M_{A}, \tan \beta\right)=(1000 \mathrm{GeV}, 3) .
\end{aligned}
$$

This high sensitivity to $\tan \beta$ is caused by the fact that the chargino contributions to the $h \rightarrow \gamma \gamma$ decay rate strongly depend on the chargino mixing, which in turn depends on

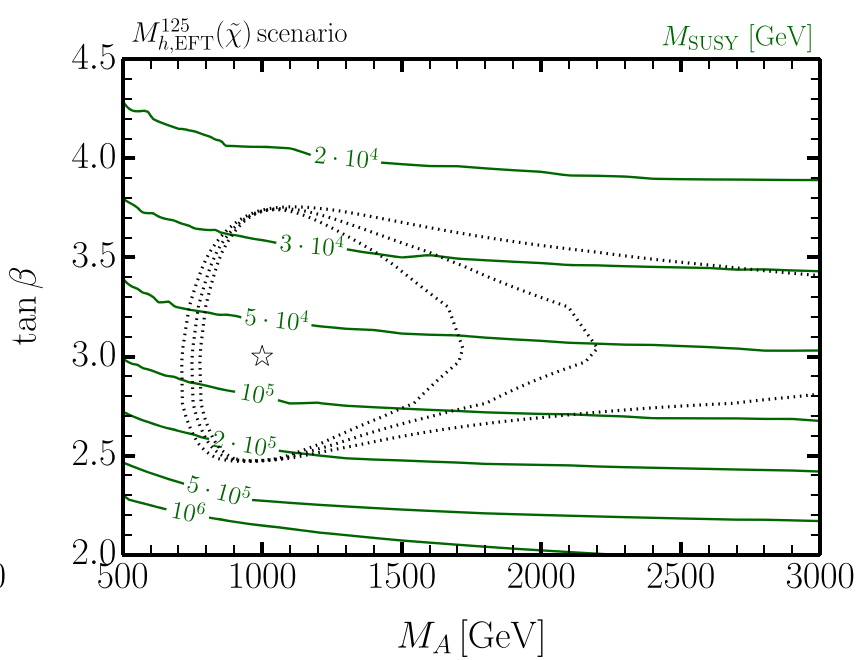

(right panel) is realized in nature. The indirect $2 \sigma$ constraints from prospective Higgs-boson signal-rate measurements at the HL-LHC and the ILC obtained in the upper panels of Fig. 12 are superimposed

$\tan \beta$. The Higgs rate measurements would complement the information from the direct searches for new particles, which in such a scenario could yield a significant excess or even a signal from the production of light electroweakinos.

The lower panels of Fig. 12 display two SM-normalized Higgs rates that are of particular importance at the HLLHC in this case: The inclusive rate for $p p \rightarrow h \rightarrow V V$ $\left(V=W^{ \pm}, Z\right)$, denoted $R_{V V}^{h}$, and the inclusive rate for $p p \rightarrow h \rightarrow \gamma \gamma$, denoted $R_{\gamma \gamma}^{h}$. The displayed results for $R_{\gamma \gamma}^{h}$ indicate that the di-photon rate is strongly influenced by loop contributions of charginos, which become large at small $\tan \beta$ values. In contrast, the $V V$ rate follows the basic trend that its decoupling behavior is mostly governed by $M_{A}$, see also the rate $R_{b b}^{V h}$ in the discussion of the $M_{h}^{125}$ scenario. The pattern of how the decoupling is approached, however, shows that for low $\tan \beta$ values slightly larger deviations from the SM value occur for the same value of $M_{A}$ than for larger $\tan \beta$. The interplay of the constraints from in particular these two rate measurements at the HL-LHC leads to the displayed shape of the allowed region that extends to the highest values of $M_{A}$ (going beyond the plotted range in the right plot) in the vicinity of $\tan \beta \sim 3$. It should be noted that for the assumed point at $M_{A}=1 \mathrm{TeV}$ (right plot) the enhancement of $R_{\gamma \gamma}^{h}$ compared to the SM value is even somewhat larger $(10 \%)$ than for the assumed point at $M_{A}=700 \mathrm{GeV}(\sim 6.5 \%$, left plot). This is because at lower $M_{A}$ values the enhancement of the $h \rightarrow b \bar{b}$ decay is stronger, which in turn suppresses the $h \rightarrow \gamma \gamma$ decay rate via its contribution to the total decay width. For the considered scenario the impact of the Higgs rate measurements at the ILC would mainly be a significant improvement of the indirect constraints on $M_{A}$. 
In Fig. 13 we show contour lines of equal $M_{\text {SUSY }}$ in the same parameter space as considered in Fig. 12. Superimposed (as dotted lines) are the expected $2 \sigma$-allowed parameter regions shown previously in Fig. 12 for the same MSSM points that we assume to be realized. MSUSY denotes the scale of all scalar fermion soft-SUSY breaking masses. As explained in Sect. 2 , in the $M_{h, \mathrm{EFT}}^{125}(\tilde{\chi})$ scenario $M_{\mathrm{SUSY}}$ is adjusted at every point in the parameter plane such that $M_{h} \simeq 125 \mathrm{GeV}$. Thus the constraints in the $\left(M_{A}, \tan \beta\right)$ parameter plane for a given assumed realization of the MSSM can be translated into a constraint on the sfermion mass scale in this scenario. As a result, if such a scenario with light electroweakinos and a rather low value of $\tan \beta$ was realized in nature, the sensitivity to $\tan \beta$ arising from the loop contributions of the light charginos to the di-photon rate could be exploited to constrain $M_{\text {SUSY }}$ to the ranges

$$
\begin{aligned}
2.3 \mathrm{TeV} \lesssim M_{\mathrm{SUSY}} \lesssim 50 \mathrm{TeV} & \text { for }\left(M_{A}, \tan \beta\right) \\
& =(700 \mathrm{GeV}, 3) \\
2.3 \mathrm{TeV} \lesssim M_{\mathrm{SUSY}} \lesssim 30 \mathrm{TeV} & \text { for }\left(M_{A}, \tan \beta\right) \\
& =(1000 \mathrm{GeV}, 3)
\end{aligned}
$$

Those indirect constraints could of course be significantly improved with the results of the direct searches for additional Higgs bosons and electroweakinos, which in the considered scenario would have good prospects for a significant excess or even a discovery.

The predicted Higgs couplings in the $M_{h, \mathrm{EFT}}^{125}(\tilde{\chi})$ scenario, parametrized in terms of $\kappa$ scale factors, are shown in Fig. 14 for the assumed MSSM points $\left(M_{A}, \tan \beta\right)=(700 \mathrm{GeV}, 3)$ (left panel) and $\left(M_{A}, \tan \beta\right)=(1 \mathrm{TeV}, 3)$ (right panel) in comparison to the anticipated $1 \sigma$ precision of the future $\kappa$ determination. In contrast to the $M_{h}^{125}$ scenario, Fig. 9, and in line with the previous discussion, because of the large loop contributions of the light charginos to the di-photon rate in the $M_{h \text {,EFT }}^{125}(\tilde{\chi})$ scenario a sizable deviation in $\kappa_{\gamma}$ is clearly visible already with the HL-LHC precision. This precision on the effective Higgs-photon-photon coupling can only mildly be improved by the ILC measurements. On the other hand, $\kappa_{b}$ and $\kappa_{\tau}$ show deviations similar to the points considered in the $M_{h}^{125}$ scenario, Fig. 9, and here the ILC measurements will be crucial to achieve a significant discrimination with respect to the SM prediction.

We now turn to the discussion of the case that a relatively large value of $\tan \beta$ could be realized in nature. For this purpose we choose a heavy Higgs-boson mass of $M_{A}=1.75 \mathrm{TeV}$. In the $M_{h}^{125}$ and $M_{h}^{125}(\tilde{\chi})$ scenarios the $\tan \beta$ value is chosen to be $\tan \beta=50$, close to the expected exclusion bound of the current $p p \rightarrow H / A \rightarrow \tau^{+} \tau^{-}$analysis [68]. For the $M_{h}^{125, \mu-}$ scenario we fix $\tan \beta=25$, close to the current indirect exclusion from Higgs rate measurements. The chosen value of $M_{A}=1.75 \mathrm{TeV}$ is a "best-case" scenario if the MSSM with a large value of $\tan \beta$ is real- ized, in the sense that it would certainly lead to a discovery of heavy Higgs bosons at the HL-LHC (see our discussion above of the projections in the different benchmark scenarios) and possibly even already in the near future.

For definiteness, we quote here the $13 \mathrm{TeV}$ signal rates of the processes $p p \rightarrow H / A \rightarrow \tau^{+} \tau^{-}$and $p p \rightarrow H / A \rightarrow$ $b \bar{b}$, whose production is completely dominated by bottomquark associated Higgs production at these parameter points. They are given by

$\sigma\left(p p \rightarrow H / A \rightarrow \tau^{+} \tau^{-}\right)=1.94 \mathrm{fb}$

in the $M_{h}^{125}$ scenario for $\left(M_{A}, \tan \beta\right)=(1.75 \mathrm{TeV}, 50)$,

$\sigma\left(p p \rightarrow H / A \rightarrow \tau^{+} \tau^{-}\right)$

$=1.51 \mathrm{fb}$ in the $M_{h}^{125}(\tilde{\chi})$

scenario for $\left(M_{A}, \tan \beta\right)=(1.75 \mathrm{TeV}, 50)$,

$\sigma\left(p p \rightarrow H / A \rightarrow \tau^{+} \tau^{-}\right)=0.49 \mathrm{fb}$ in the $M_{h}^{125, \mu-}$

scenario for $\left(M_{A}, \tan \beta\right)=(1.75 \mathrm{TeV}, 25)$,

and

$$
\begin{aligned}
& \sigma(p p \rightarrow H / A \rightarrow b \bar{b}) \\
& \quad=5.17 \mathrm{fb} \text { in the } M_{h}^{125} \\
& \quad \text { scenario for }\left(M_{A}, \tan \beta\right)=(1.75 \mathrm{TeV}, 50), \\
& \sigma(p p \rightarrow H / A \rightarrow b \bar{b}) \\
& \quad=7.16 \mathrm{fb} \text { in the } M_{h}^{125}(\tilde{\chi}) \\
& \quad \text { scenario for }\left(M_{A}, \tan \beta\right)=(1.75 \mathrm{TeV}, 50), \\
& \sigma(p p \rightarrow H / A \rightarrow b \bar{b}) \\
& \quad=11.42 \mathrm{fb} \text { in the } M_{h}^{125, \mu-} \\
& \quad \text { scenario for }\left(M_{A}, \tan \beta\right)=(1.75 \mathrm{TeV}, 25),
\end{aligned}
$$

respectively. According to our discussion in Sect. 4 we expect that the additional Higgs bosons would be first detectable in the searches in the $\tau^{+} \tau^{-}$final states, while an excess in $H / A \rightarrow b \bar{b}$ searches would at best show up in the full HL-LHC dataset (in the $M_{h}^{125, \mu-}$ scenario). It should be noted that for additional Higgs bosons in the mass range considered here the possibility that they could have a sizable branching fraction into other BSM particles should be taken seriously. For instance, for the considered parameter point in the $M_{h}^{125}(\tilde{\chi})$ scenario, the decay rates of $H$ and $A$ to electroweakinos amount to around $32 \%$. Therefore, in this scenario not only the decays of the heavy Higgs bosons to third generation leptons and quarks but also the decays into electroweakinos would be promising channels for their detection.

We now turn to the effects in the rates of the Higgs boson at $125 \mathrm{GeV}$ that would arise for the assumed parameter points with $M_{A}=1.75 \mathrm{TeV}$ and large values of $\tan \beta$. The predicted light Higgs boson couplings, presented in terms of $\kappa$ scale factors, for the three discussed MSSM scenarios are shown as Wäscheleinen-plots in Fig. 15, in comparison with 


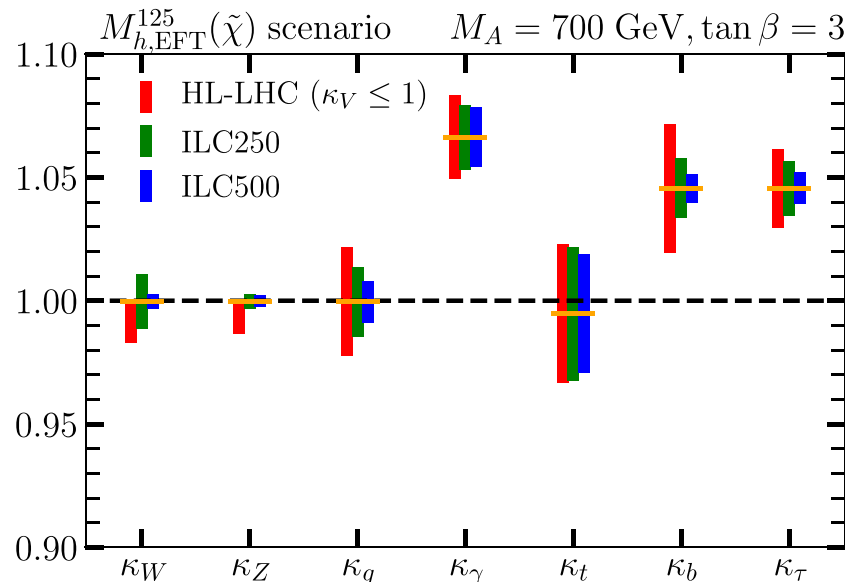

Fig. 14 Wäscheleinen-plots, using the same color coding as in Fig. 9, for the two assumed MSSM parameter points $\left(M_{A}, \tan \beta\right)=$ $(700 \mathrm{GeV}, 3)$ (left panel) and $\left(M_{A}, \tan \beta\right)=(1 \mathrm{TeV}, 3)$ (right panel) in the $M_{h, \mathrm{EFT}}^{125}(\tilde{\chi})$ scenario. The predicted Higgs couplings in the $\kappa$ frame-

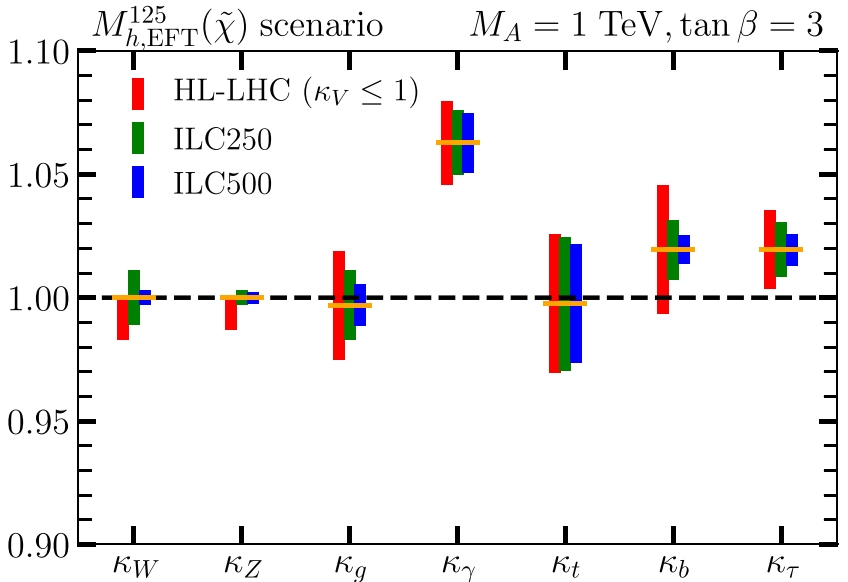

work are compared with the anticipated $1 \sigma$ precision from Higgs rate measurements, where at the HL-LHC the theoretical assumption $\kappa_{V} \leq 1$ is employed, while for the results including prospective measurements at ILC250 and ILC500 no assumption on $\kappa_{V}$ is employed
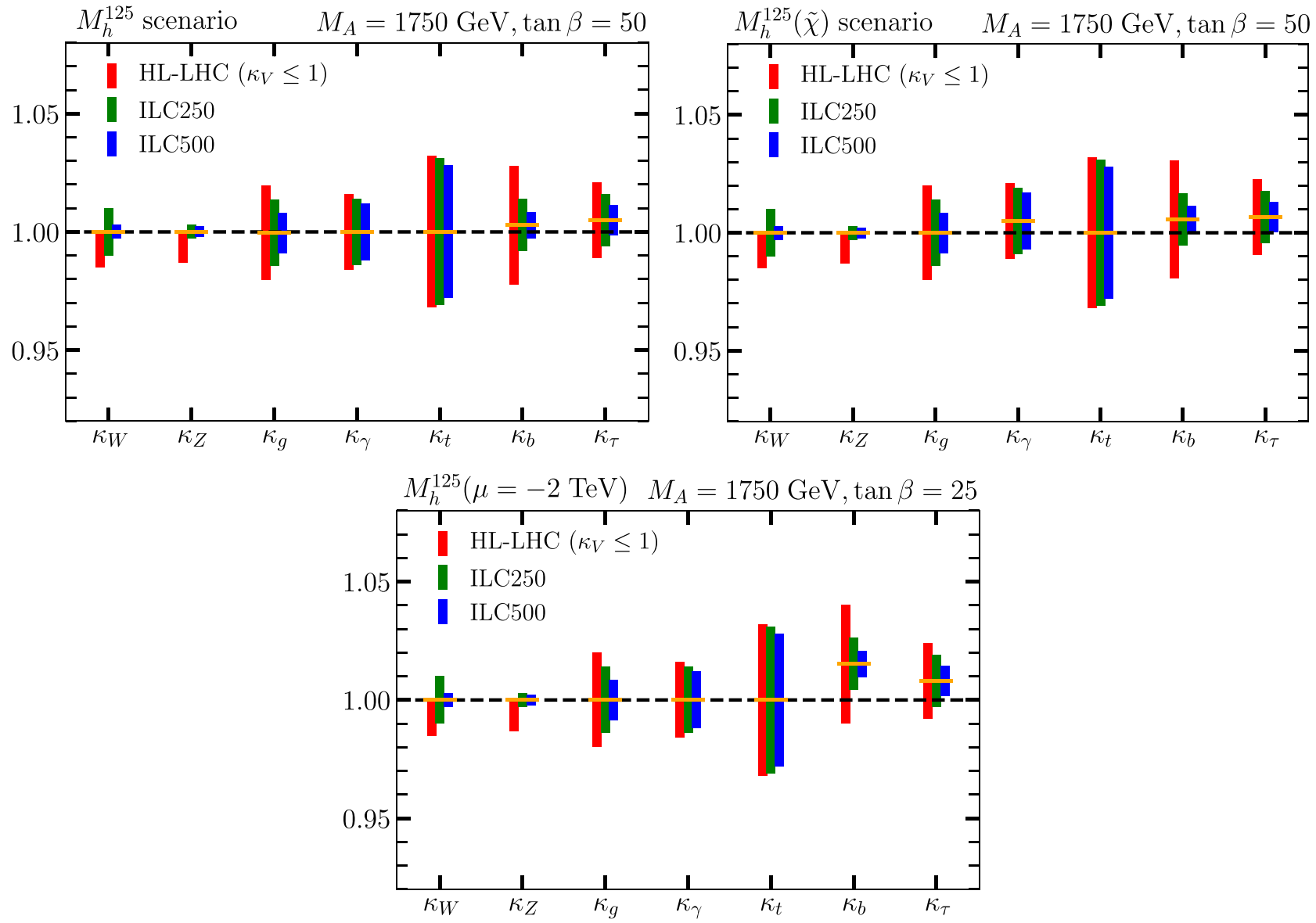

Fig. 15 Wäscheleinen-plots, using the same color coding as in Fig.9, for the following three assumed MSSM scenarios: $\left(M_{A}, \tan \beta\right)=$ $(1750 \mathrm{GeV}, 50)$ in the $M_{h}^{125}$ scenario (upper left panel), $\left(M_{A}, \tan \beta\right)=$ $(1750 \mathrm{GeV}, 50)$ in the $M_{h}^{125}(\tilde{\chi})$ scenario (upper right), and $\left(M_{A}, \tan \beta\right)=(1750 \mathrm{GeV}, 25)$ in the $M_{h}^{125, \mu-}$ scenario (lower panel).
The predicted Higgs couplings in the $\kappa$ framework are compared with the anticipated $1 \sigma$ precision from Higgs rate measurements, where at the HL-LHC the theoretical assumption $\kappa_{V} \leq 1$ is employed, while for the results including prospective measurements at ILC250 and ILC500 no assumption on $\kappa_{V}$ is employed 
Table 1 Goodness-of-fit of the SM hypothesis, $\Delta \chi_{S M}^{2}$, from a test against future signal rate measurements of the Higgs boson at $125 \mathrm{GeV}$ at the HL-LHC and in combination with ILC250 and ILC500 measurements, assuming different MSSM realizations in nature

\begin{tabular}{llrrr}
\hline MSSM scenario & Assumed $\left(M_{A}, \tan \beta\right)$ point & $\Delta \chi_{\text {SM }}^{2}$ & & \\
\cline { 3 - 5 } & & HL-LHC & ILC250 & ILC500 \\
\hline$M_{h}^{125}$ & $(700 \mathrm{GeV}, 8)$ & 22.0 & 34.3 & 50.7 \\
& $(1000 \mathrm{GeV}, 8)$ & 4.6 & 7.2 & 10.6 \\
$M_{h}^{125, \mu-}$ & $(1750 \mathrm{GeV}, 50)$ & 0.3 & 0.5 & 0.7 \\
& $(700 \mathrm{GeV}, 8)$ & 27.6 & 41.3 & 61.2 \\
$M_{h, \mathrm{EFT}}^{125}(\tilde{\chi})$ & $(1000 \mathrm{GeV}, 8)$ & 5.9 & 8.8 & 13.0 \\
$M_{h}^{125}(\tilde{\chi})$ & $(1750 \mathrm{GeV}, 25)$ & 2.5 & 3.5 & 4.2 \\
& $(700 \mathrm{GeV}, 3)$ & 113.8 & 130.7 & 152.0 \\
& $(1000 \mathrm{GeV}, 3)$ & 177.3 & 182.1 & 187.9 \\
& $(1750 \mathrm{GeV}, 50)$ & 1.2 & 1.3 & 1.7 \\
\hline
\end{tabular}

the precision of the prospective $\kappa$ determination at the HLLHC and the ILC. As expected for such a large value of $M_{A}$, corresponding to a scenario that is quite far in the decoupling region, we find that even for the relatively large chosen values of $\tan \beta$ the coupling deviations from the SM predictions are very small. Deviations at this level will be extremely challenging to resolve experimentally. The ultimate precision of ILC500 seems necessary in order to experimentally establish a significant pattern of deviations. The best chances in this context would occur if a relatively large negative value of $\mu$ was realized, as exemplified in the $M_{h}^{125, \mu-}$ scenario in Fig. 15, where an enhancement of the bottom-quark (and perhaps also tau-lepton) Yukawa coupling with respect to the SM prediction could clearly be established at the ILC. In this case the pattern and size of deviations is driven by genuine SUSY effects, i.e. beyond the THDM type II.

We summarize this discussion of MSSM points assumed to be realized in nature by giving the $\chi^{2}$ values of the SM hypothesis in these assumed scenarios for the prospective accuracies of the rate measurements at the HL-LHC and the ILC in Table 1. These $\chi^{2}$ values can be interpreted either directly as the goodness-of-fit of the SM, i.e. how well it fits the set of observations, or they can be used to perform a likelihood-ratio test that quantifies the significance of the tension between the SM prediction and the MSSM interpretation (with the assumed parameter point used as null hypothesis). Here we want to focus on the latter. As the future measurements will naturally feature statistical fluctuations, we rather refer to the $\chi^{2}$ of the SM hypothesis as $\Delta \chi_{\mathrm{SM}}^{2} \equiv \chi_{\mathrm{SM}}^{2}-\chi_{\mathrm{MSSM}}^{2}$, where in our projection study with idealized measurements we have $\chi_{\text {MSSM }}^{2}=0$ for the considered realized MSSM parameter point. In this likelihood ratio test between two simple hypotheses, with no adjustable model parameters, the levels $\Delta \chi^{2}=4$ and 9 correspond to a $2 \sigma$ and $3 \sigma$ tension, respectively, between the SM hypothesis and the MSSM hypothesis. It should be noted that this level of sensitivity does not allow one to exclude the SM hypothesis on grounds of the measurements alone, but instead only allows one to discriminate between two models. As these tensions are inferred indirectly from the signal rates of the SM-like Higgs boson, the combination with the information from direct collider searches, both regarding limits and possible hints for signals, will be crucial to firmly establish an observed pattern of BSM physics and to narrow down its possible nature.

From Table 1 we see that in the $M_{h}^{125}$ and the $M_{h}^{125, \mu-}$ scenario the HL-LHC will reveal a significant tension between the SM and the MSSM interpretation only if a realization with $M_{A}=700 \mathrm{GeV}$ is assumed. For the larger value of $M_{A}=1000 \mathrm{GeV}$, the HL-LHC can only discriminate the SM at the $2 \sigma$ level from the MSSM hypothesis, while the ILC measurements would be crucial to clearly establish a deviation from the SM. The situation is somewhat different in the $M_{h, \mathrm{EFT}}^{125}(\tilde{\chi})$ scenario at low values of $\tan \beta$, where the large enhancement of $h \rightarrow \gamma \gamma$ from loop contributions of light charginos leads to a very strong tension with the SM, already with its precise determination at the HL-LHC. In fact, such a strong deviation in the $h \rightarrow \gamma \gamma$ rate would clearly exclude the SM as null hypothesis, based solely on the goodness-of-fit, with very high significance. For the scenarios with $M_{A}=1.75 \mathrm{TeV}$ shown in Table 1 the deviations in the properties of the Higgs boson at $125 \mathrm{GeV}$ are so small that the HL-LHC accuracy for the Higgs rate measurements will yield only small shifts in $\chi^{2}$ between the SM and the MSSM. A significant distinction between the SM hypothesis and the MSSM hypothesis, with $\Delta \chi^{2}>4$, can only be achieved for the $M_{h}^{125, \mu-}$ scenario with the precision at ILC500. 


\section{Conclusions}

In this paper we have assessed the indirect sensitivity of the HL-LHC for probing extended Higgs sectors via rate measurements of the observed Higgs boson at $125 \mathrm{GeV}$, alone ${ }^{16}$ and in combination with a future $e^{+} e^{-}$Higgs factory, and we have compared the indirect sensitivity with the present LHC reach and the projected HL-LHC reach for direct searches for additional heavy Higgs bosons. For the direct searches we have taken into account present results and projected sensitivities in the $\tau^{+} \tau^{-}, b \bar{b}$ and di-Higgs $(h h)$ final states. Concerning the projections of the rate measurements of the detected Higgs boson at $125 \mathrm{GeV}$ at a future $e^{+} e^{-}$Higgs factory, we have considered for definiteness the case of the ILC since it is the currently most advanced project, and its projected accuracies are based on full detector simulations. We have performed our analysis for the specific example of the MSSM, based on benchmark scenarios that were defined in Refs. [10,11] and a new benchmark scenario, called the $M_{h}^{125, \mu-}$ scenario, which we have defined in the present paper. The $M_{h}^{125, \mu-}$ scenario is characterized by a relatively large negative value of the parameter $\mu$, namely $\mu=-2 \mathrm{TeV}$, leading to an enhancement of the Higgs-boson couplings to bottom quarks via SUSY loop contributions. This new benchmark scenario should be well suited in particular for the presentation of experimental results for the search for heavy Higgs bosons in the $H / A \rightarrow b \bar{b}$ decay channel.

Within the $M_{h}^{125}$ benchmark scenario, in which all supersymmetric particles have masses above the TeV scale such that the model at the electroweak scale resembles a THDM with MSSM relations in the Higgs sector, we find that the rate measurements at the HL-LHC could set a lower limit of about $M_{A} \gtrsim 900 \mathrm{GeV}$ in the absence of any deviation of the measured results from the SM predictions. In this scenario the indirect sensitivity from the rate measurements at the HLLHC is not sufficient to access allowed parts of the parameter space that would not be covered by the direct searches in the $\tau^{+} \tau^{-}$channel. This is in contrast to the $M_{h \text {.EFT }}^{125}$ scenario, where the region that is compatible with a prediction of about $125 \mathrm{GeV}$ for the mass of the SM-like Higgs boson extends to lower values of $\tan \beta$ around $\tan \beta \geq 1$ through a very heavy sfermion spectrum. At such low values of $\tan \beta$ the indirect HL-LHC sensitivity via the rate measurements of the state at $125 \mathrm{GeV}$ surpasses the reach of the standard search for heavy Higgs bosons in the di-tau final state. We have emphasized in this context that the search channel $H \rightarrow h h$ has the potential to cover parts of the parameter space in the low-tan $\beta$ region, and it is also of interest to exploit $H / A \rightarrow t \bar{t}$ and $A \rightarrow Z h$ searches.

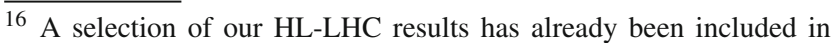
Ref. [15].
In addition to the $M_{h}^{125}$ and $M_{h \text { EFT }}^{125}$ scenarios, where all SUSY particles are relatively heavy, we have also considered the specific case of very light electroweakinos, as defined in the $M_{h}^{125}(\tilde{\chi})$ and $M_{h, \mathrm{EFT}}^{125}(\tilde{\chi})$ scenarios. These scenarios would constitute a particularly favorable case for the HLLHC, since the light charginos would give rise to large loop contributions to the $h \rightarrow \gamma \gamma$ rate (and could also be probed by direct searches for electroweakinos). In the context of a scenario of this kind, the Higgs rate measurements at the HL-LHC (assuming that they agree with the SM prediction) could be used to rule out a certain part of the plane of the two parameters $M_{2}$ and $\mu$, which together with $\tan \beta$ fix the chargino sector at tree-level. For instance, at $\tan \beta=2.5$ the HL-LHC could indirectly probe mass values of up to $\sim 255 \mathrm{GeV}$ at the $2 \sigma$ level. We have emphasized in the context of this example of a scenario where some of the SUSY particles are relatively light that the impact of direct searches for heavy Higgs bosons at the LHC can be further strengthened by supplementing the searches in the $\tau^{+} \tau^{-}$and $b \bar{b}$ final states with dedicated searches for the decays of $H$ and $A$ to BSM particles, for instance charginos, neutralinos and sleptons.

We then extended our investigations to the situation where the results from the HL-LHC are combined with prospective high-precision measurements of the Higgs signal rates at a future $e^{+} e^{-}$machine. Specifically, we considered the combination of the HL-LHC results with the results that could be achieved at the first ILC stage at $250 \mathrm{GeV}$ and $2 \mathrm{ab}^{-1}$ of data, and furthermore also the additional combination with the results achievable at the ILC at $500 \mathrm{GeV}$ with $4 \mathrm{ab}^{-1}$. In this context we did not take into account the capabilities of the ILC for detecting new light states, and we also did not attempt a combination with the prospective results from direct searches at the HL-LHC, but only commented on the possible interplay between the indirect information from the rate measurements and the results of the direct searches.

We first discussed the case where the future rate measurements at the HL-LHC and the ILC exactly agree with the SM predictions. This corresponds to an MSSM scenario that is far in the decoupling limit, where the sensitivity to variations in $M_{A}$ is small. Accordingly, the additional measurements from ILC250 and from ILC500 strengthen the indirect lower bound on $M_{A}$ from the HL-LHC measurements by only a moderate amount of about $+100 \mathrm{GeV}$ each in this scenario. It should be noted that in a realistic future analysis not all measurements would uniformly yield a push into the decoupling region, but even in case of the absence of any source of BSM physics statistical fluctuations could mimick hints for new physics. Both the gain in accuracy and the much larger set of high-precision observables that would be available from the combination of HL-LHC and ILC measurements would be instrumental to correctly interpret the observed pattern of the measurements. The analysis of scenarios with a relatively 
large negative value of $\mu$ revealed the interesting feature that the Higgs rate measurements in such a case have the potential to set an upper bound on $\tan \beta$ and/or the absolute value of $\mu$.

We furthermore investigated the question to what extent measurements at the HL-LHC and the ILC can establish significant deviations in the properties of the Higgs boson at $125 \mathrm{GeV}$ from the SM predictions via the Higgs rate measurements if a certain parameter point of the MSSM is realized in nature. In this context we also addressed the issues of how well the parameters $M_{A}$ and $\tan \beta$ can be indirectly constrained and to what extent the SM can be discriminated from the MSSM. This information, obtained on the basis of just the rate measurements of the state at $125 \mathrm{GeV}$, would be important in order to complement it with the (possibly inconclusive) information from direct searches for new physics. For this analysis we assumed that a particular parameter point of the $\left(M_{A}, \tan \beta\right)$ plane of the considered benchmark scenario is realized and we used the corresponding predictions as hypothetical results of the rate measurements performed at the HL-LHC and the ILC. Specifically we considered the $M_{A}$ values of $700 \mathrm{GeV}, 1 \mathrm{TeV}$ and $1.75 \mathrm{TeV}$ with different settings for the accompanying $\tan \beta$ value.

For the optimistic case where a value of $M_{A}=700 \mathrm{GeV}$ was realized nature, the accuracy of the rate measurements at the HL-LHC would allow one to obtain an indirect upper bound on $M_{A}$ of about $1 \mathrm{TeV}$ at the $2 \sigma$ level, which would further be strengthened with the precision measurements at the ILC. In contrast, for the case where the true value would be $M_{A}=1 \mathrm{TeV}$ the prospective accuracy of the signal strength measurements at the HL-LHC would not be sufficient to place such an indirect upper bound on $M_{A}$. This would be of relevance not only for discriminating between the SM and effects of new physics but also set a target for the direct searches for additional heavy Higgs bosons. The incorporation of the precision measurements at the ILC would not only have a strong impact regarding the distinction between the SM and the MSSM. Moreover, the much larger set of high-precision observables available from the combination of the HL-LHC with the ILC in comparison to the case of just the HL-LHC would also be crucial for disentangling the underlying nature of observed deviations from the SM. For the benchmark scenarios with light charginos the induced large loop contributions to the $h \rightarrow \gamma \gamma$ rate would make it possible, in addition to the constraints on $M_{A}$, to set tight indirect constraints on $\tan \beta$. This would be possible via the rate measurements at the HL-LHC if a low value of $\tan \beta$ was realized in nature (we considered the example of $\tan \beta=3$ ). For the case of $1.75 \mathrm{TeV}$ we considered the large values of $\tan \beta=50$ and $\tan \beta=25$, where for the former value this parameter region will be accessible via the $H / A \rightarrow \tau^{+} \tau^{-}$searches at the LHC in the near future. As expected for such a scenario that is quite far in the decoupling region, the deviations in the Higgs rates from the SM predictions are very small. A significant discrepancy from the SM could only be established with the ultimate precision of ILC500 for the $M_{h}^{125, \mu-}$ scenario.

Besides the analysis of indirect constraints in the $\left(M_{A}\right.$, $\tan \beta$ ) plane we also displayed the sensitivities in the different scenarios via "Wäscheleinen-plots" (washing line plots) showing the predicted light Higgs couplings (normalized to the SM prediction) at the assumed MSSM points in the $\kappa$ framework in comparison with the anticipated precision of the $\kappa$ determination from the prospective Higgs rate measurements at the HL-LHC and the ILC. We summarized the capabilities of the HL-LHC and the two ILC realizations to discriminate between the SM and the MSSM by providing the goodness-of-fit of the SM assuming the MSSM realizations discussed in this paper.

It should be noted that the analyses in this paper have been carried out within specific MSSM benchmark scenarios, where besides $M_{A}$ and $\tan \beta$ all other SUSY parameters are fixed to specific values by definition. This setting implies large correlations between the different Higgs rates, so that precise measurements of just a few observables already have a large impact on constraining the parameter space. As a consequence, these analyses cannot demonstrate the full potential of the entire set of high-precision observables that will be available by combining the information from the HL-LHC and the ILC. Instead, more realistically if no assumption is made on the underlying structure of the physics scenario that is realized in nature, the full breadth of precision measurements at the HL-LHC and the ILC will be crucial in order to either determine the nature of observed patterns of deviations from the SM or to set constraints on wide classes of possible extensions or alternatives to the SM. For the scenarios considered in this paper this would mean that the correlations arising from assuming the MSSM Higgs sector with the considered parameter settings could actually be tested, and results for the Higgs couplings could be obtained in a model-independent way.

Acknowledgements We thank Michael Peskin, Jürgen Reuter and Jonas Wittbrodt for interesting discussions, and Maria Cepeda, Martin Flechl, Andrew Gilbert, Marumi Kado and Lei Zhang for helpful discussions on the HL-LHC projections. H.B., T.S. and G.W. acknowledge support by the Deutsche Forschungsgemeinschaft (DFG, German Research Foundation) under Germany's Excellence Strategy - EXC 2121 "Quantum Universe" - 390833306. The work of S.H. is supported in part by the MEINCOP Spain under contract FPA2016-78022-P, in part by the Spanish "Agencia Estatal de Investigación" (AEI) and the EU "Fondo Europeo de Desarrollo Regional" (FEDER) through the project FPA2016-78022-P, in part by the "Spanish Red Consolider MultiDark" FPA2017-90566-REDC, and in part by the AEI through the grant IFT Centro de Excelencia Severo Ochoa SEV-2016-0597.

Data Availability Statement This manuscript has no associated data or the data will not be deposited. [Authors' comment: The Higgs-boson masses, cross sections and branching ratios throughout the parameter planes of our benchmark scenarios are (or will be) available in the form 
of ROOT files on the webpage of the MSSM subgroup of the LHCHXSWG].

Open Access This article is licensed under a Creative Commons Attribution 4.0 International License, which permits use, sharing, adaptation, distribution and reproduction in any medium or format, as long as you give appropriate credit to the original author(s) and the source, provide a link to the Creative Commons licence, and indicate if changes were made. The images or other third party material in this article are included in the article's Creative Commons licence, unless indicated otherwise in a credit line to the material. If material is not included in the article's Creative Commons licence and your intended use is not permitted by statutory regulation or exceeds the permitted use, you will need to obtain permission directly from the copyright holder. To view a copy of this licence, visit http://creativecomm ons.org/licenses/by/4.0/.

Funded by SCOAP ${ }^{3}$.

\section{References}

1. ATLAS Collaboration, G. Aad et al., Observation of a new particle in the search for the Standard Model Higgs boson with the ATLAS detector at the LHC. Phys. Lett. B 716, 1-29 (2012). arXiv: 1207.7214

2. CMS Collaboration, S. Chatrchyan et al., Observation of a new boson at a mass of $125 \mathrm{GeV}$ with the CMS experiment at the LHC. Phys. Lett. B 716, 30-61 (2012). arXiv:1207.7235

3. ATLAS, CMS Collaboration, G. Aad et al., Measurements of the Higgs boson production and decay rates and constraints on its couplings from a combined ATLAS and CMS analysis of the LHC pp collision data at $\sqrt{s}=7$ and $8 \mathrm{TeV}$. JHEP 08, 045 (2016). arXiv: 1606.02266

4. The ATLAS Collaboration. Combined measurements of Higgs boson production and decay using up to $80 \mathrm{fb}^{-1}$ of proton-proton collision data at $\sqrt{s}=13 \mathrm{TeV}$ collected with the ATLAS experiment. ATLAS-CONF-2019-005

5. CMS Collaboration, A.M. Sirunyan et al., Measurement and interpretation of differential cross sections for Higgs boson production at $\sqrt{s}=13$ TeV. Phys. Lett. B 792, 369-396 (2019). arXiv: 1812.06504

6. CMS Collaboration, A.M. Sirunyan et al., Combined measurements of Higgs boson couplings in proton-proton collisions at $\sqrt{s}=13 \mathrm{TeV}$. Eur. Phys. J. C 79(5), 421 (2019). arXiv: 1809.10733

7. H.P. Nilles, Supersymmetry, supergravity and particle physics. Phys. Rep. 110, 1-162 (1984)

8. H.E. Haber, G.L. Kane, The search for supersymmetry: probing physics beyond the standard model. Phys. Rep. 117, 75-263 (1985)

9. J.F. Gunion, H.E. Haber, Higgs bosons in supersymmetric models. 1. Nucl. Phys. B 272, 1 (1986) [Erratum: Nucl. Phys. B 402, 567 (1993)]

10. E. Bagnaschi et al., MSSM Higgs boson searches at the LHC: benchmark scenarios for Run 2 and beyond. Eur. Phys. J. C 79(7), 617 (2019). arXiv: 1808.07542

11. H. Bahl, S. Liebler, T. Stefaniak, MSSM Higgs benchmark scenarios for Run 2 and beyond: the low $\tan \beta$ region. Eur. Phys. J. C 79(3), 279 (2019). arXiv:1901.05933

12. O. Buchmueller et al., The CMSSM and NUHM1 after LHC Run 1. Eur. Phys. J. C 74(6), 2922 (2014). arXiv: 1312.5250

13. S. Heinemeyer, M. Mondragón, N. Tracas, G. Zoupanos, Reduction of couplings and its application in particle physics. Phys. Rep. 814, 1-43 (2019). arXiv:1904.00410
14. E. Bagnaschi et al., Likelihood analysis of the pMSSM11 in light of LHC 13-TeV data. Eur. Phys. J. C 78(3), 256 (2018). arXiv: 1710.11091

15. M. Cepeda et al., Report from working group 2. CERN Yellow Rep. Monogr. 7, 221-584 (2019). arXiv:1902.00134

16. J.F. Gunion, H.E. Haber, The CP conserving two Higgs doublet model: the approach to the decoupling limit. Phys. Rev. D 67, 075019 (2003). arXiv:hep-ph/0207010

17. M. Carena, I. Low, N.R. Shah, C.E.M. Wagner, Impersonating the standard model Higgs boson: alignment without decoupling. JHEP 04, 015 (2014). arXiv: 1310.2248

18. M. Carena, H.E. Haber, I. Low, N.R. Shah, C.E.M. Wagner, Complementarity between nonstandard Higgs boson searches and precision Higgs boson measurements in the MSSM. Phys. Rev. D 91(3), 035003 (2015). arXiv:1410.4969

19. P. Bechtle, H.E. Haber, S. Heinemeyer, O. Stål, T. Stefaniak, G. Weiglein, L. Zeune, The light and heavy Higgs interpretation of the MSSM. Eur. Phys. J. C 77(2), 67 (2017). arXiv:1608.00638

20. H.E. Haber, S. Heinemeyer, T. Stefaniak, The impact of two-loop effects on the scenario of MSSM Higgs alignment without decoupling. Eur. Phys. J. C 77(11), 742 (2017). arXiv:1708.04416

21. U. Ellwanger, C. Hugonie, A.M. Teixeira, The next-to-minimal supersymmetric standard model. Phys. Rep. 496, 1-77 (2010). arXiv:0910.1785

22. F. Domingo, G. Weiglein, NMSSM interpretations of the observed Higgs signal. JHEP 04, 095 (2016). arXiv: 1509.07283

23. P. Drechsel, L. Galeta, S. Heinemeyer, G. Weiglein, Precise predictions for the Higgs-boson masses in the NMSSM. Eur. Phys. J. C 77(1), 42 (2017). arXiv:1601.08100

24. F. Domingo, S. Heinemeyer, S. Paßehr, G. Weiglein, Decays of the neutral Higgs bosons into SM fermions and gauge bosons in the $\mathcal{C} \mathcal{P}$-violating NMSSM. Eur. Phys. J. C 78(11), 942 (2018). arXiv: 1807.06322

25. T. Biekötter, M. Chakraborti, S. Heinemeyer, A $96 \mathrm{GeV}$ Higgs boson in the N2HDM. Eur. Phys. J. C 80(1), 2 (2020). arXiv:1903.11661

26. T. Robens, T. Stefaniak, Status of the Higgs singlet extension of the standard model after LHC Run 1. Eur. Phys. J. C 75, 104 (2015). arXiv:1501.02234

27. T. Robens, T. Stefaniak, LHC benchmark scenarios for the real Higgs singlet extension of the standard model. Eur. Phys. J. C 76(5), 268 (2016). arXiv: 1601.07880

28. T. Robens, T. Stefaniak, J. Wittbrodt, Two-real-scalar-singlet extension of the SM: LHC phenomenology and benchmark scenarios. Eur. Phys. J. C 80(2), 151 (2020). arXiv:1908.08554

29. S. Heinemeyer, W. Hollik, G. Weiglein, FeynHiggs: a program for the calculation of the masses of the neutral CP even Higgs bosons in the MSSM. Comput. Phys. Commun. 124, 76-89 (2000). arXiv:hep-ph/9812320

30. S. Heinemeyer, W. Hollik, G. Weiglein, The masses of the neutral CP-even Higgs bosons in the MSSM: accurate analysis at the two loop level. Eur. Phys. J. C 9, 343-366 (1999). arXiv:hep-ph/9812472

31. G. Degrassi, S. Heinemeyer, W. Hollik, P. Slavich, G. Weiglein, Towards high precision predictions for the MSSM Higgs sector. Eur. Phys. J. C 28, 133-143 (2003). arXiv:hep-ph/0212020

32. M. Frank, T. Hahn, S. Heinemeyer, W. Hollik, H. Rzehak, G. Weiglein, The Higgs boson masses and mixings of the complex MSSM in the Feynman-diagrammatic approach. JHEP 02, 047 (2007). arXiv:hep-ph/0611326

33. T. Hahn, S. Heinemeyer, W. Hollik, H. Rzehak, G. Weiglein, Highprecision predictions for the light $\mathrm{CP}$-even Higgs boson mass of the minimal supersymmetric standard model. Phys. Rev. Lett. 112(14), 141801 (2014). arXiv:1312.4937 
34. H. Bahl, W. Hollik, Precise prediction for the light MSSM Higgs boson mass combining effective field theory and fixed-order calculations. Eur. Phys. J. C 76(9), 499 (2016). arXiv:1608.01880

35. H. Bahl, S. Heinemeyer, W. Hollik, G. Weiglein, Reconciling EFT and hybrid calculations of the light MSSM Higgs-boson mass. Eur. Phys. J. C 78(1), 57 (2018). arXiv: 1706.00346

36. H. Bahl, W. Hollik, Precise prediction of the MSSM Higgs boson masses for low $\mathrm{M}_{A}$. JHEP 07, 182 (2018). arXiv: 1805.00867

37. H. Bahl, T. Hahn, S. Heinemeyer, W. Hollik, S. Paßehr, H. Rzehak, G. Weiglein, Precision calculations in the MSSM Higgsboson sector with FeynHiggs 2.14. Comput. Phys. Commun. 249, 107099 (2020). https://doi.org/10.1016/j.cpc.2019.107099

38. H. Bahl, S. Heinemeyer, W. Hollik, G. Weiglein, Theoretical uncertainties in the MSSM Higgs boson mass calculation. Eur. Phys. J. C 80(6), 497 (2020). https://doi.org/10.1140/epjc/ s10052-020-8079-3

39. R.V. Harlander, S. Liebler, H. Mantler, SusHi: a program for the calculation of Higgs production in gluon fusion and bottom-quark annihilation in the Standard Model and the MSSM. Comput. Phys. Commun. 184, 1605-1617 (2013). arXiv:1212.3249

40. R.V. Harlander, S. Liebler, H. Mantler, SusHi Bento: beyond NNLO and the heavy-top limit. Comput. Phys. Commun. 212, 239-257 (2017). arXiv: 1605.03190

41. R. Harlander, P. Kant, Higgs production and decay: analytic results at next-to-leading order QCD. JHEP 12, 015 (2005). arXiv:hep-ph/0509189

42. R.V. Harlander, W.B. Kilgore, Next-to-next-to-leading order Higgs production at hadron colliders. Phys. Rev. Lett. 88, 201801 (2002). arXiv:hep-ph/0201206

43. R.V. Harlander, W.B. Kilgore, Production of a pseudoscalar Higgs boson at hadron colliders at next-to-next-to leading order. JHEP 10, 017 (2002). arXiv:hep-ph/0208096

44. C. Anastasiou, C. Duhr, F. Dulat, E. Furlan, T. Gehrmann, F. Herzog, B. Mistlberger, Higgs boson gluonFfusion production beyond threshold in $\mathrm{N}^{3}$ LO QCD. JHEP 03, 091 (2015). arXiv:1411.3584

45. C. Anastasiou, C. Duhr, F. Dulat, E. Furlan, F. Herzog, B. Mistlberger, Soft expansion of double-real-virtual corrections to Higgs production at $\mathrm{N}^{3}$ LO. JHEP 08, 051 (2015). arXiv: 1505.04110

46. C. Anastasiou, C. Duhr, F. Dulat, E. Furlan, T. Gehrmann, F. Herzog, A. Lazopoulos, B. Mistlberger, High precision determination of the gluon fusion Higgs boson cross-section at the LHC. JHEP 05, 058 (2016). arXiv:1602.00695

47. G. Degrassi, P. Slavich, NLO QCD bottom corrections to Higgs boson production in the MSSM. JHEP 11, 044 (2010). arXiv: 1007.3465

48. G. Degrassi, S. Di Vita, P. Slavich, NLO QCD corrections to pseudoscalar Higgs production in the MSSM. JHEP 08, 128 (2011). arXiv:1107.0914

49. G. Degrassi, S. Di Vita, P. Slavich, On the NLO QCD corrections to the production of the heaviest neutral Higgs scalar in the MSSM. Eur. Phys. J. C 72, 2032 (2012). arXiv: 1204.1016

50. S. Actis, G. Passarino, C. Sturm, S. Uccirati, NLO electroweak corrections to Higgs boson production at hadron colliders. Phys. Lett. B 670, 12-17 (2008). arXiv:0809.1301

51. M. Spira, A. Djouadi, D. Graudenz, P. Zerwas, Higgs boson production at the LHC. Nucl. Phys. B 453, 17-82 (1995). arXiv:hep-ph/9504378

52. M. Bonvini, A.S. Papanastasiou, F.J. Tackmann, Resummation and matching of b-quark mass effects in $b \bar{b} H$ production. JHEP 11, 196 (2015). arXiv: 1508.03288

53. M. Bonvini, A.S. Papanastasiou, F.J. Tackmann, Matched predictions for the $b \bar{b} H$ cross section at the 13 TeV LHC. JHEP 10, 053 (2016). arXiv: 1605.01733

54. S. Forte, D. Napoletano, M. Ubiali, Higgs production in bottomquark fusion in a matched scheme. Phys. Lett. B 751, 331-337 (2015). arXiv: 1508.01529
55. S. Forte, D. Napoletano, M. Ubiali, Higgs production in bottomquark fusion: matching beyond leading order. Phys. Lett. B 763, 190-196 (2016). arXiv:1607.00389

56. T. Banks, Supersymmetry and the quark mass matrix. Nucl. Phys. B 303, 172-188 (1988)

57. L.J. Hall, R. Rattazzi, U. Sarid, The top quark mass in supersymmetric SO(10) unification. Phys. Rev. D 50, 7048-7065 (1994). arXiv:hep-ph/9306309

58. R. Hempfling, Yukawa coupling unification with supersymmetric threshold corrections. Phys. Rev. D 49, 6168-6172 (1994)

59. M. Carena, M. Olechowski, S. Pokorski, C.E.M. Wagner, Electroweak symmetry breaking and bottom-top Yukawa unification. Nucl. Phys. B 426, 269-300 (1994). arXiv:hep-ph/9402253

60. M. Carena, D. Garcia, U. Nierste, C.E.M. Wagner, Effective Lagrangian for the $\bar{t} b H^{+}$interaction in the MSSM and charged Higgs phenomenology. Nucl. Phys. B 577, 88-120 (2000). arXiv:hep-ph/9912516

61. M. Carena, D. Garcia, U. Nierste, C.E.M. Wagner, $b \rightarrow s \gamma$ and supersymmetry with large $\tan \beta$. Phys. Lett. B 499, 141-146 (2001). arXiv:hep-ph/0010003

62. M. Carena, S. Heinemeyer, O. Stål, C.E.M. Wagner, G. Weiglein, MSSM Higgs boson searches at the LHC: benchmark scenarios after the discovery of a Higgs-like particle. Eur. Phys. J. C 73(9), 2552 (2013). arXiv: 1302.7033

63. LHC Higgs Cross Section Working Group Collaboration, D. de Florian et al., Handbook of LHC Higgs cross sections: 4. Deciphering the nature of the Higgs sector. arXiv: 1610.07922

64. D. Noth, M. Spira, Higgs boson couplings to bottom quarks: two-loop supersymmetry-QCD corrections. Phys. Rev. Lett. 101, 181801 (2008). arXiv:0808.0087

65. D. Noth, M. Spira, Supersymmetric Higgs Yukawa couplings to bottom quarks at next-to-next-to-leading order. JHEP 06, 084 (2011). arXiv:1001.1935

66. L. Mihaila, C. Reisser, $\mathcal{O}\left(\right.$ alph $\left._{s}^{2}\right)$ corrections to fermionic Higgs decays in the MSSM. JHEP 08, 021 (2010). arXiv:1007.0693

67. A. Georges et al., ATLAS Collaboration, Search for heavy neutral Higgs bosons produced in association with $b$-quarks and decaying into $b$-quarks at $\sqrt{s}=13 \mathrm{TeV}$ with the ATLAS detector. Phys. Rev. D 102(3), 032004 (2020). https://doi.org/10.1103/ PhysRevD.102.032004

68. CMS Collaboration, A.M. Sirunyan et al., Search for additional neutral MSSM Higgs bosons in the $\tau \tau$ final state in protonproton collisions at $\sqrt{s}=13 \mathrm{TeV}$. JHEP 09, 007 (2018). arXiv: 1803.06553

69. P. Bechtle, O. Brein, S. Heinemeyer, G. Weiglein, K.E. Williams, HiggsBounds: confronting arbitrary Higgs sectors with exclusion bounds from LEP and the Tevatron. Comput. Phys. Commun. 181, 138-167 (2010). arXiv:0811.4169

70. P. Bechtle, O. Brein, S. Heinemeyer, G. Weiglein, K.E. Williams, HiggsBounds 2.0.0: confronting neutral and charged Higgs sector predictions with exclusion bounds from LEP and the Tevatron. Comput. Phys. Commun. 182, 2605-2631 (2011). arXiv: 1102.1898

71. P. Bechtle, O. Brein, S. Heinemeyer, O. Stal, T. Stefaniak, G. Weiglein, K. Williams, Recent developments in HiggsBounds and a preview of HiggsSignals. PoS CHARGED2012, 024 (2012). arXiv: 1301.2345

72. P. Bechtle, O. Brein, S. Heinemeyer, O. Stål, T. Stefaniak, G. Weiglein, K.E. Williams, HiggsBounds - 4: improved tests of extended Higgs sectors against exclusion bounds from LEP, the Tevatron and the LHC. Eur. Phys. J. C 74(3), 2693 (2014). arXiv: 1311.0055

73. P. Bechtle, S. Heinemeyer, O. Stal, T. Stefaniak, G. Weiglein, Applying exclusion likelihoods from LHC searches to extended Higgs sectors. Eur. Phys. J. C 75(9), 421 (2015). arXiv:1507.06706 
74. P. Bechtle, D. Dercks, S. Heinemeyer, T. Klingl, T. Stefaniak, G. Weiglein, J. Wittbrodt, HiggsBounds-5: testing Higgs sectors in the LHC $13 \mathrm{TeV}$ era (2000). arXiv:2006.06007

75. P. Bechtle, S. Heinemeyer, O. Stål, T. Stefaniak, G. Weiglein, HiggsSignals: confronting arbitrary Higgs sectors with measurements at the Tevatron and the LHC. Eur. Phys. J. C 74(2), 2711 (2014). arXiv: 1305.1933

76. P. Bechtle, S. Heinemeyer, T. Klingl, T. Stefaniak, G. Weiglein, J. Wittbrodt, HiggsSignals-2: probing new physics with precision Higgs measurements in the LHC $13 \mathrm{TeV}$ era (to be published)

77. G. Aad et al., ATLAS Collaboration, Combined measurements of Higgs boson production and decay using up to $80 \mathrm{fb}^{-1}$ of protonproton collision data at $\sqrt{s}=13 \mathrm{TeV}$ collected with the ATLAS experiment. Phys. Rev. D 101(1), 012002 (2020). https://doi.org/ 10.1103/PhysRevD.101.012002

78. CMS Collaboration, A.M. Sirunyan et al., Measurements of properties of the Higgs boson decaying to a $\mathrm{W}$ boson pair in $\mathrm{pp}$ collisions at $\sqrt{s}=13 \mathrm{TeV}$. Phys. Lett. B 791, 96 (2019). arXiv: 1806.05246

79. CMS Collaboration, Measurements of properties of the Higgs boson in the four-lepton final state in proton-proton collisions at $\sqrt{s}=13$ TeV. CMS-PAS-HIG-19-001

80. CMS Collaboration, Measurements of Higgs boson production via gluon fusion and vector boson fusion in the diphoton decay channel at $\sqrt{s}=13 \mathrm{TeV}$. CMS-PAS-HIG-18-029

81. CMS Collaboration, A.M. Sirunyan et al., Search for the Higgs boson decaying to two muons in proton-proton collisions at $\sqrt{s}=13$ TeV. Phys. Rev. Lett. 122(2), 021801 (2019). arXiv: 1807.06325

82. CMS Collaboration, Measurement of Higgs boson production and decay to the $\tau \tau$ final state. CMS-PAS-HIG-18-032

83. CMS Collaboration, A.M. Sirunyan et al., Evidence for the Higgs boson decay to a bottom quark-antiquark pair. Phys. Lett. B 780, 501-532 (2018). arXiv: 1709.07497

84. CMS Collaboration, A.M. Sirunyan et al., Inclusive search for a highly boosted Higgs boson decaying to a bottom quark-antiquark pair. Phys. Rev. Lett. 120(7), 071802 (2018). arXiv:1709.05543

85. CMS Collaboration, Measurement of $t \bar{t} H$ production in the $H \rightarrow$ $b \bar{b}$ decay channel in $41.5 \mathrm{fb}^{-1}$ of proton-proton collision data at $\sqrt{s}=13 \mathrm{TeV}$. CMS-PAS-HIG-18-030

86. CMS Collaboration, A.M. Sirunyan et al., Evidence for associated production of a Higgs boson with a top quark pair in final states with electrons, muons, and hadronically decaying $\tau$ leptons at $\sqrt{s}=13$ TeV. JHEP 08, 066 (2018). arXiv: 1803.05485

87. CMS Collaboration, Measurement of the associated production of a Higgs boson with a top quark pair in final states with electrons, muons and hadronically decaying $\tau$ leptons in data recorded in 2017 at $\sqrt{s}=13 \mathrm{TeV}$. CMS-PAS-HIG-18-019

88. M. Carena, S. Heinemeyer, C.E.M. Wagner, G. Weiglein, MSSM Higgs boson searches at the Tevatron and the LHC: impact of different benchmark scenarios. Eur. Phys. J. C 45, 797-814 (2006). arXiv:hep-ph/0511023

89. CMS Collaboration, Projection of the Run 2 MSSM H $\rightarrow \tau \tau$ limits for the high-luminosity LHC. CMS-PAS-FTR-18-017

90. A. Adhikary, S. Banerjee, R. Kumar Barman, B. Bhattacherjee, Resonant heavy Higgs searches at the HL-LHC. JHEP 09, 068 (2019). arXiv: 1812.05640

91. CMS Collaboration, A.M. Sirunyan et al., Combination of searches for Higgs boson pair production in proton-proton collisions at $\sqrt{s}=13$ TeV. Phys. Rev. Lett. 122(12), 121803 (2019). arXiv: 1811.09689

92. A. Djouadi, J. Ellis, J. Quevillon, Interference effects in the decays of spin-zero resonances into $\gamma \gamma$ and $t \bar{t}$. JHEP 07, 105 (2016). arXiv: 1605.00542
93. M. Carena, Z. Liu, Challenges and opportunities for heavy scalar searches in the $t \bar{t}$ channel at the LHC. JHEP 11, 159 (2016). arXiv: 1608.07282

94. B. Hespel, F. Maltoni, E. Vryonidou, Signal background interference effects in heavy scalar production and decay to a top-anti-top pair. JHEP 10, 016 (2016). arXiv: 1606.04149

95. D. Buarque Franzosi, E. Vryonidou, C. Zhang, Scalar production and decay to top quarks including interference effects at NLO in QCD in an EFT approach. JHEP 10, 096 (2017). arXiv: 1707.06760

96. W. Bernreuther, P. Galler, C. Mellein, Z.G. Si, P. Uwer, Production of heavy Higgs bosons and decay into top quarks at the LHC. Phys. Rev. D 93(3), 034032 (2016). arXiv:1511.05584

97. W. Bernreuther, P. Galler, Z.-G. Si, P. Uwer, Production of heavy Higgs bosons and decay into top quarks at the LHC. II: topquark polarization and spin correlation effects. Phys. Rev. D 95(9), 095012 (2017). arXiv: 1702.06063

98. A. Djouadi, J. Ellis, A. Popov, J. Quevillon, Interference effects in $t \bar{t}$ production at the LHC as a window on new physics. JHEP 03, 119 (2019). arXiv:1901.03417

99. ATLAS Collaboration, M. Aaboud et al., Search for Heavy Higgs bosons $A / H$ decaying to a top quark pair in $p p$ collisions at $\sqrt{s}=8 \mathrm{TeV}$ with the ATLAS detector. Phys. Rev. Lett. 119(19), 191803 (2017). arXiv: 1707.06025

100. CMS Collaboration, Search for heavy Higgs bosons decaying to a top quark pair in proton-proton collisions at $\sqrt{s}=13 \mathrm{TeV}$. CMS-PAS-HIG-17-027

101. A. Djouadi, L. Maiani, G. Moreau, A. Polosa, J. Quevillon, V. Riquer, The post-Higgs MSSM scenario: Habemus MSSM? Eur. Phys. J. C 73, 2650 (2013). arXiv: 1307.5205

102. A. Djouadi, J. Quevillon, The MSSM Higgs sector at a high $M_{S U S Y}$ : reopening the low $\tan \beta$ regime and heavy Higgs searches. JHEP 10, 028 (2013). arXiv:1304.1787

103. L. Maiani, A.D. Polosa, V. Riquer, Bounds to the Higgs sector masses in minimal supersymmetry from LHC data. Phys. Lett. B 724, 274-277 (2013). arXiv:1305.2172

104. A. Djouadi, L. Maiani, A. Polosa, J. Quevillon, V. Riquer, Fully covering the MSSM Higgs sector at the LHC. JHEP 06, 168 (2015). arXiv: 1502.05653

105. S. Liebler, M. Mühlleitner, M. Spira, M. Stadelmaier, The hMSSM approach for Higgs self-couplings revisited. Eur. Phys. J. C 79(1), 65 (2019). arXiv: 1810.10979

106. A. Aboubrahim, P. Nath, Naturalness, the hyperbolic branch, and prospects for the observation of charged Higgs bosons at high luminosity LHC and $27 \mathrm{TeV}$ LHC. Phys. Rev. D 98(9), 095024 (2018). arXiv: 1810.12868

107. J. de Blas et al., Higgs Boson studies at future particle colliders. JHEP 01, 139 (2020). https://doi.org/10.1007/JHEP01(2020)139

108. A. Freitas, S. Heinemeyer, et al., Theoretical uncertainties for electroweak and Higgs-boson precision measurements at FCCee. arXiv: 1906.05379

109. ATLAS Collaboration, M. Aaboud et al., Search for additional heavy neutral Higgs and gauge bosons in the ditau final state produced in $36 \mathrm{fb}^{-1}$ of pp collisions at $\sqrt{s}=13 \mathrm{TeV}$ with the ATLAS detector. JHEP 01, 055 (2018). arXiv: 1709.07242

110. G. Aad et al., ATLAS Collaboration, Search for heavy Higgs bosons decaying into two tau leptons with the ATLAS detector using $p p$ collisions at $\sqrt{s}=13 \mathrm{TeV}$. Phys. Rev. Lett. 125(5), 051801 (2020). https://doi.org/10.1103/PhysRevLett. 125.051801

111. ATLAS Collaboration, ATLAS searches for MSSM Higgs bosons decaying into SUSY cascades. ATL-PHYS-PUB-2009-079, ATLCOM-PHYS-2009-086

112. M. Bisset, J. Li, N. Kersting, R. Lu, F. Moortgat, S. Moretti, Fourlepton LHC events from MSSM Higgs boson decays into neutralino and chargino pairs. JHEP 08, 037 (2009). arXiv:0709.1029 
113. C. Charlot, R. Salerno, Y. Sirois, Observability of the heavy neutral SUSY Higgs bosons decaying into neutralinos. J. Phys. G 34, N1-N12 (2007)

114. A. Arbey, M. Battaglia, F. Mahmoudi, Supersymmetric heavy Higgs bosons at the LHC. Phys. Rev. D 88(1), 015007 (2013). arXiv: 1303.7450

115. CMS Collaboration, G. Bayatian et al., CMS technical design report, volume II: physics performance. J. Phys. G 34(6), 9951579 (2007)

116. N. Craig, F. D'Eramo, P. Draper, S. Thomas, H. Zhang, The hunt for the rest of the Higgs bosons. JHEP 06, 137 (2015). arXiv: 1504.04630

117. G. Belanger, D. Ghosh, R. Godbole, S. Kulkarni, Light stop in the MSSM after LHC Run 1. JHEP 09, 214 (2015). arXiv: 1506.00665

118. R.K. Barman, B. Bhattacherjee, A. Chakraborty, A. Choudhury, Study of MSSM heavy Higgs bosons decaying into charginos and neutralinos. Phys. Rev. D 94(7), 075013 (2016). arXiv: 1607.00676

119. S. Baum, K. Freese, N.R. Shah, B. Shakya, NMSSM Higgs boson search strategies at the LHC and the mono-Higgs signature in particular. Phys. Rev. D 95(11), 115036(2017). arXiv:1703.07800

120. S. Profumo, T. Stefaniak, L. Stephenson Haskins, The not-sowell tempered neutralino. Phys. Rev. D 96(5), 055018 (2017). arXiv: 1706.08537

121. S. Kulkarni, L. Lechner, Characterizing simplified models for heavy Higgs decays to supersymmetric particles. arXiv: 1711.00056

122. E. Arganda, V. Martin-Lozano, A.D. Medina, N. Mileo, Potential discovery of staus through heavy Higgs boson decays at the LHC. JHEP 09, 056 (2018). arXiv:1804.10698

123. S. Gori, Z. Liu, B. Shakya, Heavy Higgs as a portal to the supersymmetric electroweak sector. JHEP 04, 049 (2019). arXiv: 1811.11918

124. A. Adhikary, B. Bhattacherjee, R.M. Godbole, N. Khan, S. Kulkarni, Searching for heavy Higgs in supersymmetric final states at the LHC. arXiv:2002.07137

125. J. Liu, N. McGinnis, C.E.M. Wagner, X.-P. Wang, Searching for the Higgsino-Bino sector at the LHC. JHEP 09, 73 (2020). https:// doi.org/10.1007/JHEP09(2020)073

126. S. Heinemeyer, C. Schappacher, Heavy Higgs decays into Sfermions in the complex MSSM: a full one-loop analysis. Eur. Phys. J. C 75(5), 198 (2015). arXiv: 1410.2787

127. S. Heinemeyer, C. Schappacher, Higgs decays into charginos and neutralinos in the complex MSSM: a full one-loop analysis. Eur. Phys. J. C 75(5), 230 (2015). arXiv:1503.02996

128. R.K. Barman, G. Belanger, B. Bhattacherjee, R. Godbole, G. Mendiratta, D. Sengupta, Invisible decay of the Higgs boson in the context of a thermal and nonthermal relic in MSSM. Phys. Rev. D 95(9), 095018 (2017). arXiv: 1703.03838

129. G. Pozzo, Y. Zhang, Constraining resonant dark matter with combined LHC electroweakino searches. Phys. Lett. B 789, 582-591 (2019). arXiv: 1807.01476
130. K. Wang, J. Zhu, Funnel annihilations of light dark matter and the invisible decay of the Higgs boson. Phys. Rev. D 101(9), 095028 (2020). arXiv:2003.01662

131. R.K. Barman, G. Bélanger, B. Bhattacherjee, R. Godbole, D. Sengupta, X. Tata, Current bounds and future prospects of light neutralino dark matter in NMSSM. arXiv:2006.07854

132. X. Cid Vidal et al., Report from working group 3. CERN Yellow Rep. Monogr. 7, 585-865 (2019). arXiv:1812.07831

133. T. Biekotter, S. Heinemeyer, C. Munoz, Precise prediction for the Higgs-boson masses in the $\mu \nu$ SSM. Eur. Phys. J. C 78(6), 504 (2018). arXiv: 1712.07475

134. P. Drechsel, G. Moortgat-Pick, G. Weiglein, Sensitivity of the ILC to light Higgs masses, in International Workshop on Future Linear Collider (LCWS2017) Strasbourg, France, October 23-27, 2017, 2018. arXiv: 1801.09662

135. International Large Detector Concept Group Collaboration, Y. Wang, Search for light scalars produced in association with a Z boson at the $250 \mathrm{GeV}$ stage of the ILC. PoS ICHEP2018, 630 (2019)

136. International Large Detector Concept Group Collaboration, Y. Wang, J. List, M. Berggren, Search for extra scalars produced in association with muon pairs at the ILC, in International Workshop on Future Linear Colliders (LCWS 2018) Arlington, Texas, USA, October 22-26, 2018, 2019. arXiv:1902.06118

137. T. Biekotter, S. Heinemeyer, C. Munoz, Precise prediction for the Higgs-boson masses in the $\mu \nu$ SSM with three righthanded neutrino superfields. Eur. Phys. J. C 79(8), 667 (2019). arXiv: 1906.06173

138. P. Bechtle, S. Heinemeyer, O. Stål, T. Stefaniak, G. Weiglein, Probing the standard model with Higgs signal rates from the Tevatron, the LHC and a future ILC. JHEP 11, 039 (2014). arXiv: 1403.1582

139. K. Desch, E. Gross, S. Heinemeyer, G. Weiglein, L. Zivkovic, LHC / LC interplay in the MSSM Higgs sector. JHEP 09, 062 (2004). arXiv:hep-ph/0406322

140. LHC/LC Study Group Collaboration, G. Weiglein et al., Physics interplay of the LHC and the ILC. Phys. Rep. 426, 47-358 (2006). arXiv:hep-ph/0410364

141. T. Barklow, K. Fujii, S. Jung, R. Karl, J. List, T. Ogawa, M.E. Peskin, J. Tian, Improved formalism for precision Higgs coupling fits. Phys. Rev. D 97(5), 053003 (2018). arXiv:1708.08912 